Submitted to The Astronomical Journal

Preprint typeset using $\mathrm{LAT}_{\mathrm{E}} \mathrm{X}$ style emulateapj v. 11/26/03

\title{
THE TEAM KECK TREASURY REDSHIFT SURVEY OF THE GOODS-NORTH FIELD ${ }^{1}$
}

\author{
Gregory D. Wirth ${ }^{2}$, Christopher N. A. Willmer ${ }^{3,4}$, Paola Amico ${ }^{2}$, Frederic H. Chaffee $^{2}$, Robert W. Goodrich $^{2}$, \\ Shui Kwok ${ }^{2}$, James E. Lyke ${ }^{2}$, Jeff A. Mader ${ }^{2}$, Hien D. Tran ${ }^{2}$, Amy J. Barger ${ }^{5,6}$, Lennox L. Cowie ${ }^{5}$, \\ Peter CapaK ${ }^{5}$, Alison L. Coil ${ }^{7}$, Michael C. Cooper ${ }^{7}$, Al Conrad ${ }^{2}$, Mard Davis $^{7}, \mathrm{~S}_{\text {. M. Faber }}^{3}$, Esther M. Hu ${ }^{5}$, \\ David C. Koo ${ }^{3}$, David Le Mignant ${ }^{2}$, Jeffrey A. Newman ${ }^{7}$, Antoinette Songaila ${ }^{5}$ \\ Submitted to The Astronomical Journal
}

\begin{abstract}
We report the results of an extensive imaging and spectroscopic survey in the GOODS-North field completed using DEIMOS on the Keck II telescope. Observations of 2018 targets in a magnitudelimited sample of 2911 objects to $R_{A B}=24.4$ yield secure redshifts for a sample of 1440 galaxies and AGN plus 96 stars. In addition to redshifts and associated quality assessments, our catalog also includes photometric and astrometric measurements for all targets detected in our $R$-band imaging survey of the GOODS-North region. We investigate various sources of incompleteness and find the redshift catalog to be $53 \%$ complete at its limiting magnitude. The median redshift of $z=0.65$ is lower than in similar deep surveys because we did not select against low-redshift targets.

Comparison with other redshift surveys in the same field, including a complementary Hawaii-led DEIMOS survey, establishes that our velocity uncertainties are as low as $\sigma \approx 40 \mathrm{~km} \mathrm{~s}^{-1}$ for red galaxies and that our redshift confidence assessments are accurate. The distributions of rest-frame magnitudes and colors among the sample agree well with model predictions out to and beyond $z=1$. We will release all survey data, including extracted 1-D and sky-subtracted 2-D spectra, thus providing a sizable and homogeneous database for the GOODS-North field which will enable studies of large scale structure, spectral indices, internal galaxy kinematics, and the predictive capabilities of photometric redshifts.

Subject headings: Galaxies: distances and redshifts - galaxies: photometry
\end{abstract}

\section{INTRODUCTION}

In 1995, the Hubble Deep Field (HDF) became the most well-studied extragalactic area in all of observational astronomy. Extremely deep optical exposures taken with the Hubble Space Telescope (HST) were eventually joined by additional imaging and spectroscopic observations from many observatories covering all wavelengths (cf. Ferguson. Dickinson. \& Williams (2000) and references therein). This combined dataset - which was ultimately expanded to include a complementary field in the southern sky - painted an extraordinarily detailed picture of a "blank" region of sky which turned out to be teeming with galaxies, providing astronomers with an exquisite window into the high-redshift universe. The result was a series of important advances in the properties of distant galaxies and large-scale structure in the universe. Many of these key discoveries depended critically upon knowledge of galaxy redshifts,

\footnotetext{
1 Based in part on data obtained at the W. M. Keck Observatory, which is operated as a scientific partnership among the California Institute of Technology, the University of California, and NASA, and was made possible by the generous financial support of the W. M. Keck Foundation.

${ }_{2}$ W. M. Keck Observatory, Kamuela, HI 96743; zee@keck.hawaii.edu.

${ }^{3}$ University of California Observatories/Lick Observatory, Department of Astronomy \& Astrophysics, University of California, Santa Cruz, CA 95064.

4 On leave from Observatório Nacional, Brazil.

5 Institute for Astronomy, University of Hawaii, 2680 Woodlawn Drive, Honolulu, HI 96822.

6 Department of Astronomy, University of Wisconsin-Madison, 475 North Charter Street, Madison, WI 53706.

7 Department of Astronomy, University of California, Berkeley, CA 94720
}

the vast majority of which were obtained by a variety of observers using the LRIS spectrograph (Oke et al. 1995) on the twin $10 \mathrm{~m}$ telescopes of the W. M. Keck Observatory (Cohen et al. 1996; Cowie et al. 1996; Steidel et al. 1996; Lowenthal et al. 1997; Phillips et al. 1997; Moustakas et al. 1997; Cohen et al. 2000; Cohen 2001; Dawson et al. 2001).

Nearly ten years later, attention has returned to the northern HDF field (HDF-N) via an ambitious plan to use a new generation of ground- and space-based instruments for probing the distant universe in even greater detail. The Great Observatories Origins Deep Survey (GOODS; Dickinson et al. (2001)) project involves a coordinated effort by three of NASA's Great Observatories to obtain ultra-deep images of two selected fields in the optical (via the HST Advanced Camera for Surveys), infrared (with the Spitzer Space Telescope), and X-ray (using Chandra) wavelength regimes. These two fields include a northern target area coincident with (but significantly larger than) the HDF-N plus a similarly-sized southern field coincident with the Chandra Deep FieldSouth. Again, intensive ground-based followup observations in all wavelength regimes are underway to exploit this high-resolution space-based imaging. High-quality spectra of the many galaxies in the GOODS fields will, as before, be essential to understand both large-scale structure and galaxy evolution within these regions.

Keck remains the most powerful northern-hemisphere observatory for completing deep surveys of faint galaxies. Its efficient new DEIMOS spectrograph (Faber et al. 2003) on the Keck II telescope was designed specifically for acquiring multislit spectroscopy of over 100 faint galaxies at once, and with its $16^{\prime}$ field of view is ide- 
ally suited to survey the $10^{\prime} \times 16^{\prime}$ GOODS-North (hereafter GOODS-N) field. Presented with this unique opportunity to make an important contribution to the community, the WMKO Director and scientific staff elected to complete a deep DEIMOS survey of this region and committed to releasing the resulting data to the community as rapidly as possible. We report herein the results of this "Team Keck" Treasury Redshift Survey (hereafter, TKRS) in the GOODS-N field. Our survey is complemented by the Hawaii-led Keck/DEIMOS survey in GOODS-N described in the accompanying report (Cowie et al. 2004), and also by a Gemini-North survey completed with GMOS (Dickinson et al. 2004).

In \$2 of this report we review the construction of the photometric catalog of the GOODS-N field on which we based our slitmask designs. Section 3 discusses the acquisition and reduction of the spectra and the determination of redshifts. Section 4 concerns the redshift catalog, including the effects of target characteristics on the sample's completeness, the comparison of our survey with others in the same area, the accuracy of our redshifts and quality assessments, and the accuracy of photometric redshifts in this field. Section 15 presents the redshift and rest-frame color distribution for our targets, and $\$ 6$ concludes the paper.

\section{PHOTOMETRIC CATALOG}

To ensure timely access to a catalog of astrometric positions and approximate magnitudes for completing the spectroscopy, we generated our own independent catalog of sources in the GOODS-N field. We acquired a series of $300 \mathrm{~s} R$-band exposures using DEIMOS in imaging mode on Keck II during the night of 2002 Dec 30 (UT), and complemented these with additional images obtained on 2003 Feb 02 (UT) to complete coverage of the full ACS imaging region. The exposures were offset and dithered to compensate for the gaps in the DEIMOS CCD mosaic.

All images were bias subtracted and gain corrected, then flatfielded with sky flats generated from the set of 14 images acquired in this field. We employed an automated procedure to detect cosmic rays on each image and ignored the affected pixels in all subsequent processing. The mean sky level on each image was measured via an iterative sigma clipping algorithm and subtracted to eliminate sky brightness variations from the data set. Next, we used DEIMOS images of an astrometric field to derive a distortion correction map which we applied to the images of the GOODS-N field. This allowed us to shift and co-add the science images, yielding a mosaic image that covered the entire $10^{\prime} \times 16^{\prime}$ first-epoch ACS imaging field. For this survey, we only considered the portion of this mosaic image coincident with the first epoch $H S T$ GOODS-N observations. We obtained a first-order plate solution for this mosaic using several stars measured in the USNO astrometric catalog (Monet et al. 2003).

To generate our target catalog we used the SExTRACTOR package (Bertin \& Arnouts 1996) to detect positive fluctuations at least $20 \mathrm{px}$ in size with a threshold of 1.0 times the RMS sky noise. Because of vignetting near the edges of the DEIMOS imaging field of view, the final mosaic image contains a few regions with spurious detections. These bogus objects are generally very faint, and most were excluded from the final catalogue because they lie below the faint magnitude cutoff of the sample.
Although the USNO catalog provided an effective firstorder astrometric solution for the GOODS-N mosaic image, the small number of sources available in this field limited the accuracy of the solution. The resultant RMS positional uncertainty of approximately 0.5 is a significant fraction of the $1^{\prime \prime}$ slit width for spectroscopy, and hence a more accurate solution was needed to produce acceptable slitmask designs. In order to improve the object positions, we used a catalogue of objects in this region kindly provided by the SDSS team (York et al. 2000) which produced a list of 130 stars common to both catalogs. This permitted us to refine the astrometric solution and obtain a final RMS residual of $\sim 0$ "' 13 , more than adequate for our needs. Comparing our derived astrometric coordinates to those obtained independently by Capak et al. (2003), we find no measureable offset in $(\alpha, \delta)$ and a dispersion of $\sigma=0$ "' 40 , indicating excellent agreement. Our coordinates agree less well in absolute zero point with those Giavalisco et al. (2004) obtained from $H S T$ imaging, against which the dispersion is $00^{\prime \prime} 23$ but the offsets are $\Delta \alpha=0^{\prime \prime} 00$ and $\Delta \delta=0^{\prime \prime} 38$; this suggests a slight error in absolute astrometric positions for the ACS catalog. For several saturated stars which we wanted to use for rough slitmask alignment purposes, we derived estimated celestial coordinates positions visually by estimating the $(\alpha, \delta)$ coordinates for the location at the convergence points of the diffraction spikes.

The unsaturated stars also served as photometric calibrators. The magnitudes we used were MAG_BEST calculated by SExTRACTOR, which are designed to estimate the total light. Since only $r^{\prime}$ magnitudes were available from the SDSS database, we were unable to include any color terms in our photometric calibration. For the 130 stars shared between the TKRS and SDSS catalogs, the RMS difference is $\Delta R=0.36 \mathrm{mag}$. The final magnitudes are in the AB system (Oke 1974), roughly equivalent to the SDSS $r^{\prime}$. Figure 1 compares the SDSS $r^{\prime}$ and DEIMOS $R$ instrumental response functions.

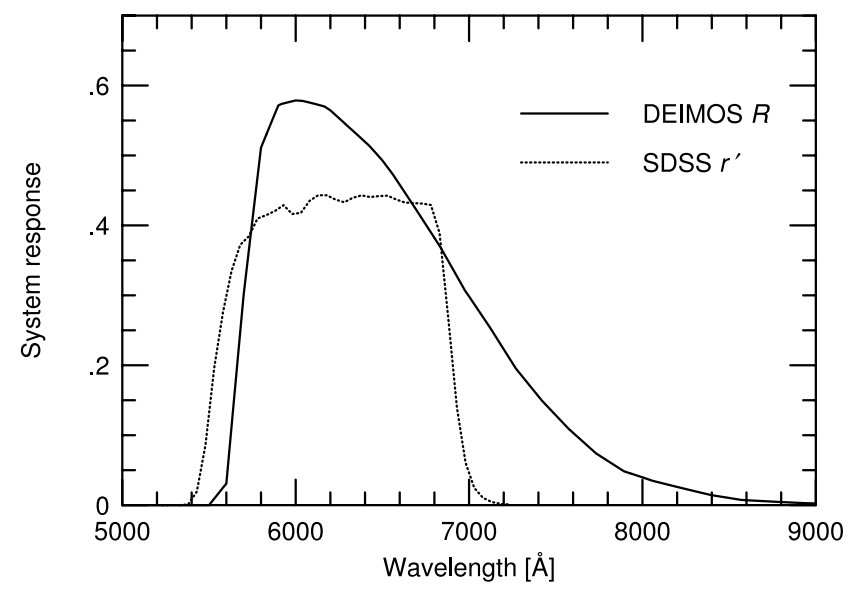

FIG. 1.- System (telescope, filter, and detector) response curves of $R$ band filter used in the DEIMOS imaging (solid line) and the SDSS $r^{\prime}$ filter (dotted line). The $r^{\prime}$ filter is part of the system devised to calibrate the SDSS survey (Smith et al. 2002), and is shown here to illustrate the differences in bandpass of the filters. Note that in the actual calibration of the TKRS catalogue, the $r$-band data from the SDSS DR1 release were used. 


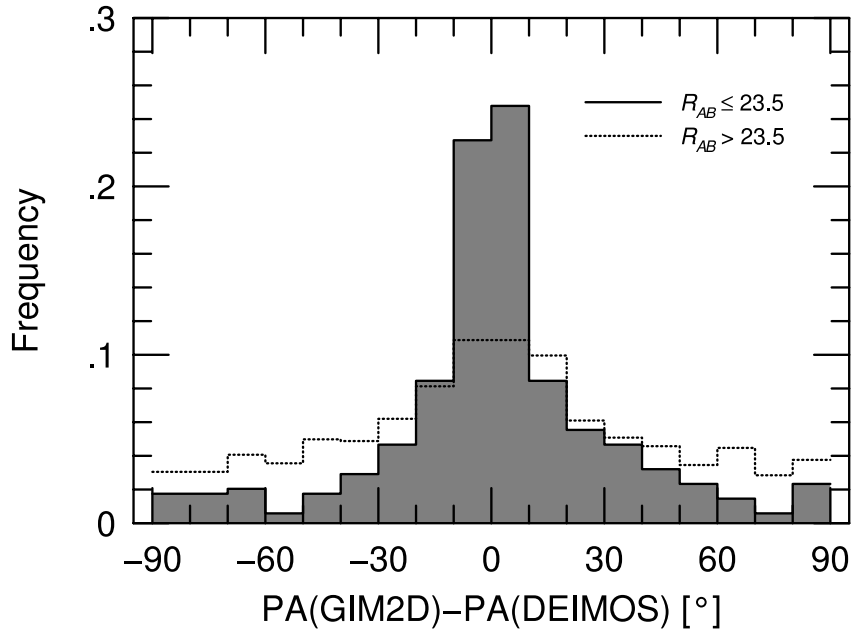

FIG. 2.- Distribution of the difference in position angle measured from ground-based DEIMOS $R$-band images vs. HST WFPC2 $I$-band images in the HDF and Flanking Fields (Marleau \& Simard 1998; Simard et al. 2002), binned in $10^{\circ}$ intervals. The solid histogram represents brighter galaxies with $R_{A B} \leq 23.5$, while the solid line represents the position angle differences for galaxies fainter than 23.5. Both bright and faint galaxies show a peak at position angle differences at $0^{\circ}$, but whereas this peak is well defined for the brighter galaxies, for fainter ones the smoother distribution indicates a poor correlation.

SEXTRACTOR also provides for each object an estimate of its orientation as represented by the major axis position angle. This information can be used to orient spectroscopic slits to the major axis of the galaxy and thus obtain both improved $S / N$ spectra and more accurate rotation curves. We compared the position angles derived from the DEIMOS mosaic image with independent measurements ${ }^{8}$ obtained from HST images by the UCSC DEEP1 team in the Hubble Deep Field and Flanking Fields using two-dimensional two-parameter fits to the images (Marleau \& Simard 1998; Simard et al. 2002). A histogram comparing the difference in position angles using $10^{\circ}$ bins is shown in Fig. 22 The solid histogram shows the distribution measured for galaxies with $R \leq 23.5$, while the dotted line represents the fainter galaxies. Both distributions have been normalized by the total number of galaxies in each subsample. Although both distributions are peaked at values close to $0^{\circ}$, indicating that the two datasets generally agree, the peak is far more pronounced for the brighter galaxy sample. We conclude that the position angles measured by SEXTRACTOR are adequate for the brighter galaxies.

\section{SPECTROSCOPY}

\subsection{Slitmask design}

The selection of objects for spectroscopic observations followed roughly the same guidelines as used by the DEEP2 Redshift Survey (Davis et al. 2003), with one major difference: no attempt was made to use brightness, color, or other information to exclude stars and lowerredshift galaxies from the sample. All objects brighter than the limiting magnitude were potential spectroscopic

\footnotetext{
${ }^{8}$ Data available at http://archive.deep.ucolick.org
}

targets, regardless of whether their appearance was stellar or galactic. At the magnitude limit of this survey the number of stars relative to the number of galaxies is small, so that the adopted strategy avoids excluding AGN-dominated galaxies.

DEIMOS uses slitmasks to record over 100 broadband spectra at once. The limiting magnitude of the spectroscopic sample, the number of available nights and uniformity of wavelength coverage were all considered in designing slitmasks for our survey. The magnitude cutoff was set at $R \leq 24.4$ in order to provide sufficient source density per slitmask, yielding a sample of 2911 candidate targets for inclusion on masks. To improve consistency of wavelength coverage, objects were preferentially placed near the center of the $4^{\prime}$ available in the spatial direction on each DEIMOS mask. Automated slitmask design software developed for the DEEP2 survey then selected appropriate targets from the catalog to produce a set of 18 slitmasks, each displaced from the next by $\sim 0$.'33, to cover the GOODS-N field. The resulting distribution in equatorial coordinates of the 2018 objects in the spectroscopic sample is shown in Fig. 3

All slits were tilted by a minimum amount with respect to the CCD rows in order to improve the wavelength sampling of the spectra; the slight offset between adjacent columns in the spectra serves to dither the night sky lines and thus permit a more accurate reconstruction of the background spectrum. For objects exhibiting significant elongation in the DEIMOS images, slits were typically aligned with the major axis to improve both $S / N$ and rotational velocity measurements. For closely spaced objects, slit position angles were chosen such that the spectra of both objects were recorded. However, slits were never tilted by more than $\pm 30^{\circ}$ from the nominal position angle of the slitmask.

\subsection{Observations}

Spectroscopic observations of the GOODS-N field were completed using DEIMOS on the Keck II telescope during early-to-mid 2003 as detailed in Table 1 Each observation used the $600 \mathrm{l} \mathrm{mm}^{-1}$ grating blazed at $7500 \AA$, with the GG455 order-blocking filter eliminating all flux below $4550 \AA$. The central wavelength was set at $7200 \AA$, providing nominal spectral coverage of $4600-9800 \AA$ at a FWHM resolution of $\Gamma \approx 3.5 \AA$ A. Eleven masks were observed during time allocated to the WMKO Director, and the remaining seven were generously completed by the DEEP2 Redshift Survey Team. Each slitmask was observed for a total on-source integration time of $3600 \mathrm{~s}$, broken up into $3 \times 1200 \mathrm{~s}$ integrations to allow rejection of cosmic rays. No dithering was performed between exposures because of the use of tilted slits and because the minor fringing pattern present in DEIMOS images is sufficiently corrected by the use of flat field images.

To enable wavelength calibration and the removal of instrumental signatures, calibration data consisting of three internal quartz flats and a single arc lamp spectrum (Kr, Ar, Ne, Xe) were obtained for each mask in the daytime; the DEIMOS flexure compensation system ensured that these calibration images matched the science frames to better than $\pm 0.25 \mathrm{px}$. The transparency and seeing conditions on Mauna Kea varied from fair to excellent during the observations, affecting the resultant data quality. 


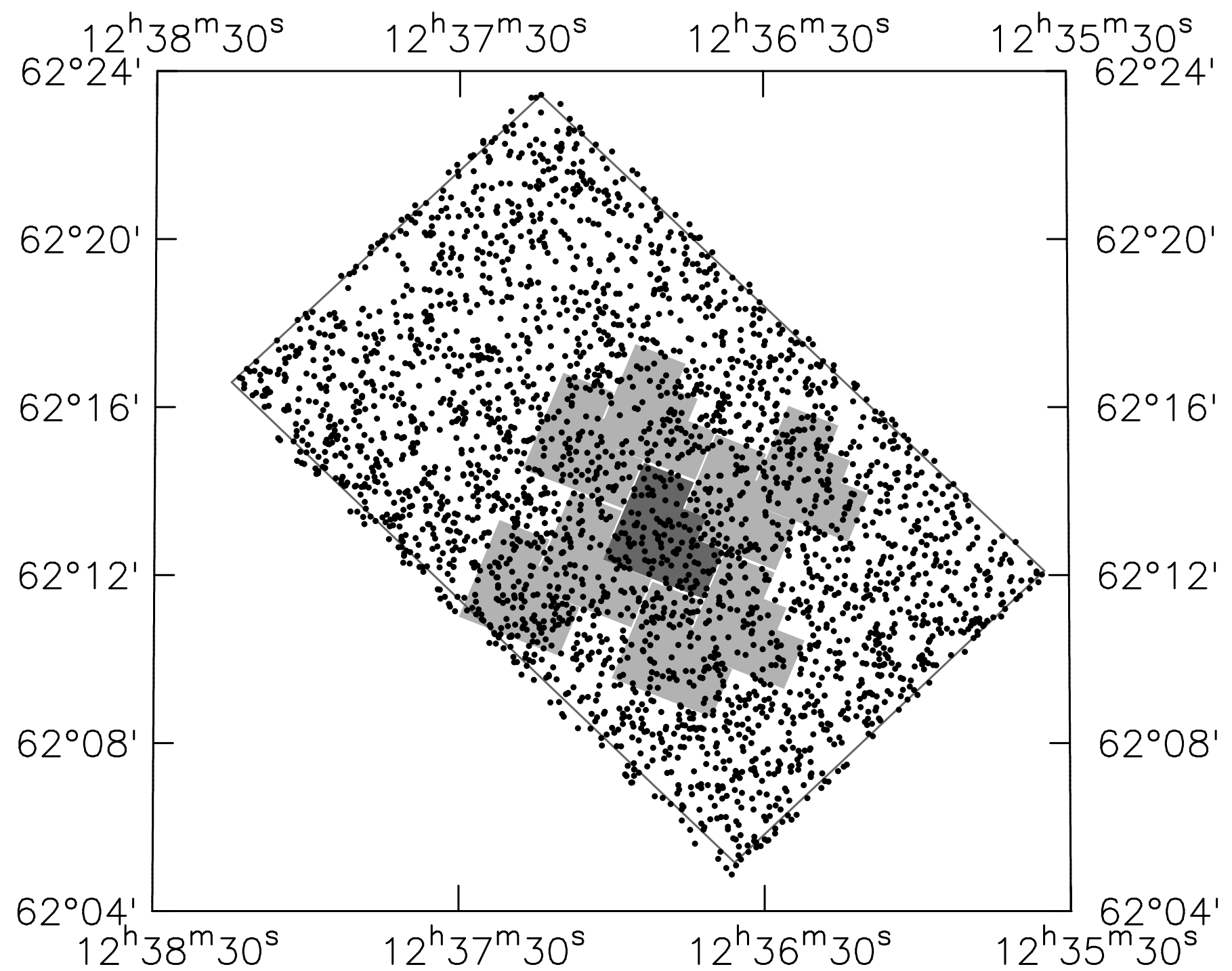

FIG. 3.- Projected sky distribution of objects in the DEIMOS spectroscopic survey sample. Shaded areas indicate the Hubble Deep Field (dark grey) and the flanking fields (light grey). The outline indicates approximately the first-epoch HST ACS GOODS-N imaging survey region.

\subsection{Data reduction}

All spectra were extracted using the fully-automated pipeline developed by the DEEP2 Redshift Survey Team (Newman et al. 2004) and generously made available to us. Each spectral exposure was divided by its corresponding flatfield image to remove small amounts of CCD fringing. The three exposures for each mask were then combined to yield an image cleaned of cosmic rays. For each slitlet, a 2-D sky-subtracted spectrum is obtained by modeling the night-sky with a B-spline and subtracting it from the combined 2-D spectrum. Wavelength calibration was achieved by fitting to the arc lamp emission lines. The sky-subtracted spectrum of the target was produced by summing over the rows containing the flux from the object as predicted by the tabulated position relative to the slit endpoints.

\subsection{Redshift determination}

The first phase of measuring redshifts involved fully automated processing of each spectrum using the same algorithm as the SDSS (Strauss et al. 2002), which calcu- lates for each lag position (i.e., redshift) the best representation of the source spectrum through the use of a set of template eigenspectra Glazebrook, Offer \& Deelev 1998). These templates include several types of stars, a galaxy absorption spectrum, a galaxy emission-line spectrum, and an AGN spectrum. The best fit is determined from the minimum $\chi^{2}$ in the redshift-lag and eigenspectra parameter space. The ten best fits are stored in the 1-D image header.

In the second phase, all spectra were visually inspected by at least two team members, who would select both a redshift and a corresponding quality code; objects for which no unambiguous redshift could be determined were also noted. Due to low $S / N$ spectra or to poor matches between the spectra of the templates and some program objects, the automated redshift determination algorithm sometimes selected an incorrect redshift (Newman, private communication). However, all redshifts reported herein were agreed upon by two reviewers, and can thus be considered secure. The adopted redshift quality codes (hereafter, $Q$; see Table 21) are the same as used by the 
Table 1. Slitmask Observation Data

\begin{tabular}{ccccrrrr}
\hline \hline Mask & $\begin{array}{c}\text { Obs. Date } \\
(\mathrm{UT})\end{array}$ & $\begin{array}{c}\alpha^{\mathrm{a}} \\
(\mathrm{J} 2000)\end{array}$ & $\begin{array}{c}\delta^{\mathrm{a}} \\
(\mathrm{J} 2000)\end{array}$ & $\begin{array}{c}\mathrm{PA}^{\mathrm{b}} \\
\left({ }^{\circ}\right)\end{array}$ & $N_{o}{ }^{\mathrm{c}}$ & $N_{z}{ }^{\mathrm{d}}$ & $\begin{array}{c}F_{z}{ }^{\mathrm{e}} \\
(\%)\end{array}$ \\
\hline 1 & 2003 May 02 & 123630.81 & 621701.9 & 44 & 117 & 72 & 62 \\
2 & 2003 May 02 & 123633.44 & 621633.9 & 44 & 118 & 91 & 77 \\
3 & 2003 May 02 & 123636.99 & 621620.2 & 44 & 118 & 94 & 80 \\
4 & 2003 May 03 & 123639.63 & 621552.2 & 44 & 111 & 97 & 87 \\
5 & 2003 May 03 & 123643.18 & 621538.5 & 44 & 112 & 99 & 88 \\
6 & 2003 May 03 & 123645.81 & 621510.5 & 44 & 103 & 94 & 91 \\
7 & 2003 May 04 & 123649.37 & 621456.9 & 44 & 103 & 69 & 67 \\
8 & 2003 May 05 & 123652.00 & 621428.8 & 44 & 105 & 80 & 76 \\
9 & 2003 May 06 & 123655.56 & 621415.2 & 44 & 110 & 89 & 81 \\
10 & 2003 Mar 26 & 123658.19 & 621347.1 & 44 & 105 & 62 & 59 \\
11 & 2003 Mar 26 & 123701.75 & 621333.5 & 44 & 114 & 88 & 77 \\
12 & 2003 Mar 26 & 123704.38 & 621305.5 & 44 & 112 & 93 & 83 \\
13 & 2003 Mar 26 & 123707.93 & 621251.8 & 44 & 102 & 73 & 72 \\
14 & 2003 Mar 26 & 123710.57 & 621223.8 & 44 & 118 & 75 & 64 \\
15 & 2003 May 28 & 123714.12 & 621210.1 & 44 & 120 & 104 & 87 \\
16 & 2003 May 28 & 123716.75 & 621142.1 & 44 & 125 & 98 & 78 \\
17 & 2003 May 29 & 123720.31 & 621128.5 & 44 & 116 & 86 & 74 \\
18 & 2003 May 29 & 123719.86 & 621121.3 & -136 & 109 & 72 & 66 \\
& & & & & & & \\
\hline
\end{tabular}

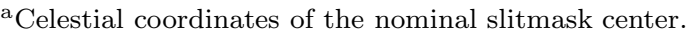

${ }^{\mathrm{b}}$ Position angle of the slitmask; individual slit orientations vary.

${ }^{\mathrm{c}}$ Number of objects per mask; note that a slit may contain multiple objects.

${ }^{\mathrm{d}}$ Number of secure redshifts $(Q=-1,3$, or 4$)$ measured per mask.

ePercentage of objects per mask yielding secure redshifts.

DEEP2 team (Newman et al. 2004). We consider the accuracy of these quality codes in 4.5

The results from the visual inspections were then collated and discrepant cases noted and reassessed by one individual. Here, "discrepant" means that one observer assigned a $Q$ value of 3 or 4 (corresponding to a "secure" redshift) whereas another would select $Q=2$ or less, or that two observers would assign secure $Q$ values but discrepant redshifts. The latter cases resulted when spectra of two different galaxies overlapped in the same slit.

The distribution of redshift quality codes among the 2018 observed targets is shown in Table 2 The number of secure redshift measurements $(Q=-1,3,4)$ is 1536 . The $Q=-2$ objects are technically not counted as failures; hence, the overall efficiency is $77 \%$ (1536/1987), which is comparable to the success rate of DEEP2 and similar redshift surveys (e.g., Cowie et al. (2004)).

\section{REDSHIFT CATALOG}

\subsection{Data}

The measurements resulting from our survey are presented in Table [6 a portion of which is reproduced here. This table appears in its entirety in the electronic version of the Journal and also on the website devoted to the survey ${ }^{9}$. Photometric and spectroscopic data for all targets detected in our imaging survey appear here, in addition to alternate redshifts from other surveys when available. Redshifts for targets in our survey are only given when they are classified as secure $(Q=-1,3,4)$. Also available on the survey website is the master table of measurements which combines the results of this survey with those of the parallel effort by Cowie et al. (2004). Users of these data should note that the tabulated redshifts for our survey currently lack a heliocentric correction; an adjustment will be applied in future

\footnotetext{
9 http://www2.keck.hawaii.edu/science/tksurvey
}

releases, thus changing the zero point for the redshifts by no more than a few tens of $\mathrm{km} \mathrm{s}^{-1}$.

\subsection{Sample completeness}

In the present section we examine the systematics of the present sample as a function of location in the DEIMOS field of view, apparent magnitude, color, and surface brightness. The number of objects placed on each mask appears in Table 1 which also shows both the number of targets for which secure redshifts were obtained $(N(Q=-1,3,4))$ and the success fraction per mask. On average, each mask contained about 112 objects, of which 76 (68\%) produced secure redshifts. However, these numbers do not take into account the $Q=-2$ objects representing failures due to instrumental effects, so these numbers understate the survey's redshift measuring efficiency.

Spatial effects. - Figures 4 and 5 depict the distribution of galaxies as a function of the DEIMOS mosaic $x$ and $y$ coordinates, respectively. The figures compare the distribution of all targets (dark grey histograms) to those which yielded secure redshifts (light grey histogram). In Fig. 4 the periodic depressions result in part from a lack of slits placed in the inter-CCD gap regions (a product of the slitmask design) and in part due to the decreased success rate for spectra taken acquired too close to the gaps and ruined. The decrease in number of objects at the edges of the DEIMOS mosaic $y$ (shorter) axis is clearly seen in Fig. 5 and this is probably an effect caused by the slitmask design algorithm. For galaxies and stars located at the edges there is only one chance of being placed on masks, in contrast to objects further from the edge.

We show the spatial distribution of objects for which redshifts were not successfully measured in Fig. 6] Objects which produced no measurable spectrum $(Q=-2)$ because of instrumental effects are shown as circles. 
Table 2. TKRS Redshift Quality Distribution

\begin{tabular}{|c|c|c|c|c|c|c|}
\hline$Q^{\mathrm{a}}$ & Definition & $N_{o b j}{ }^{\mathrm{b}}$ & $F_{o b j}{ }^{\mathrm{c}}$ & $N_{z o} \mathrm{~d}$ & $N_{\text {agree }} \mathrm{e}$ & $\begin{array}{l}F_{\text {agree }}^{\mathrm{f}} \\
(\%)\end{array}$ \\
\hline 4 & $\begin{array}{l}\text { Very secure redshift }(P>99 \%) \text {; at least two spectral features } \\
\text { identified }\end{array}$ & 1180 & 58.5 & 154 & 145 & 94.2 \\
\hline 3 & $\begin{array}{l}\text { Secure redshift }(P>95 \%) \text {; one strong line and another weak feature } \\
\text { identified or single wide line, typically }[\mathrm{O} \text { II] } \lambda 3727 \text { blend }\end{array}$ & 260 & 12.9 & 83 & 76 & 91.6 \\
\hline 2 & $\begin{array}{l}\text { Uncertain redshift; signal is present but no unambiguous spectral line } \\
\text { identified }\end{array}$ & 268 & 13.3 & 69 & 26 & 37.7 \\
\hline 1 & No redshift; $S / N$ too poor & 183 & 9.1 & 28 & 1 & 3.6 \\
\hline-1 & Star & 96 & 4.8 & 50 & 49 & 98.0 \\
\hline-2 & No redshift measured because of instrumental artifacts in spectrum & 31 & 1.5 & 6 & 0 & 0.0 \\
\hline
\end{tabular}

a TKRS redshift quality category.

${ }^{\mathrm{b}}$ Number of objects in TKRS catalog for this category.

${ }^{\mathrm{c}}$ Fraction (percentage) of objects in TKRS catalog for this category.

dNumber of TKRS objects in this category which have independent redshift measurements for comparison.

eNumber of TKRS objects for which the independent redshifts agree to within $|\Delta z| \leq 0.002$.

${ }^{\mathrm{f}}$ Fraction (percentage) of TKRS objects for which the independent redshifts agree to within $|\Delta z| \leq 0.002$.

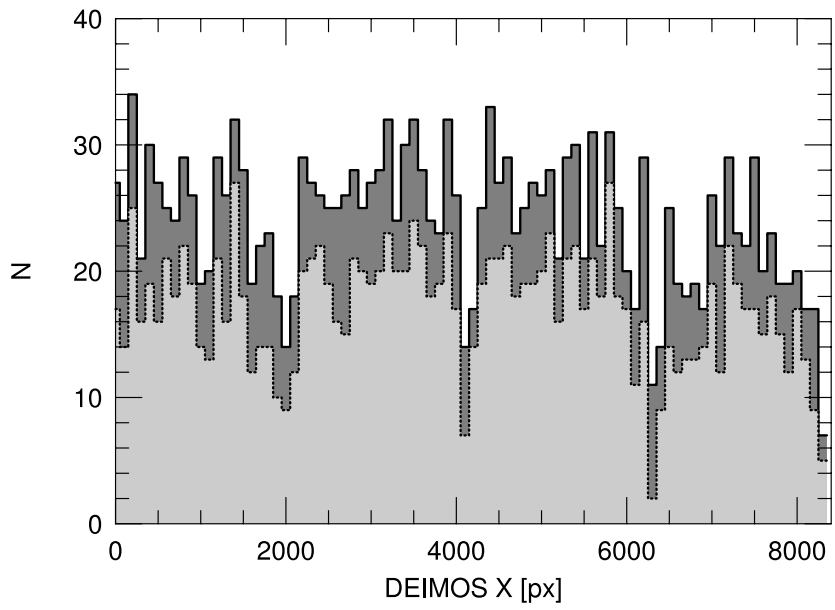

FIG. 4.- Number of targets placed on masks (dark grey histogram), and number of successful redshift measures (light grey) as a function of the DEIMOS catalog $X$ coordinate, which is parallel to the long axis of the GOODS-N field. As explained in the text, the depressions at $X=2000,4000$ and 6000 correspond to the gaps between CCDs.

Since these failures are primarily due to spectra falling on the gaps between CCDs in the DEIMOS detector mosaic or to objects placed near the mask edges, they cluster in several lines across the GOODS-N field. Targets whose redshifts are either completely unknown $(Q=1)$ or highly uncertain $(Q=2)$ are distributed randomly across the field, as expected.

Magnitude effects. - Our survey's sampling and success rates as a function of apparent magnitude are shown in Fig. [7and Table 3 For each 0.5 mag bin, the shaded histogram represents the differential quantity and the solid line (when present) indicates the integrated completeness down to that magnitude. Figure Z(a) shows the number of available targets in our photometric catalog of the GOODS-N field, with the number in the rightmost bin reduced by the effect of our $R_{A B}=24.4$ cutoff. Panel (b) shows the fraction of targets we observed spectro-

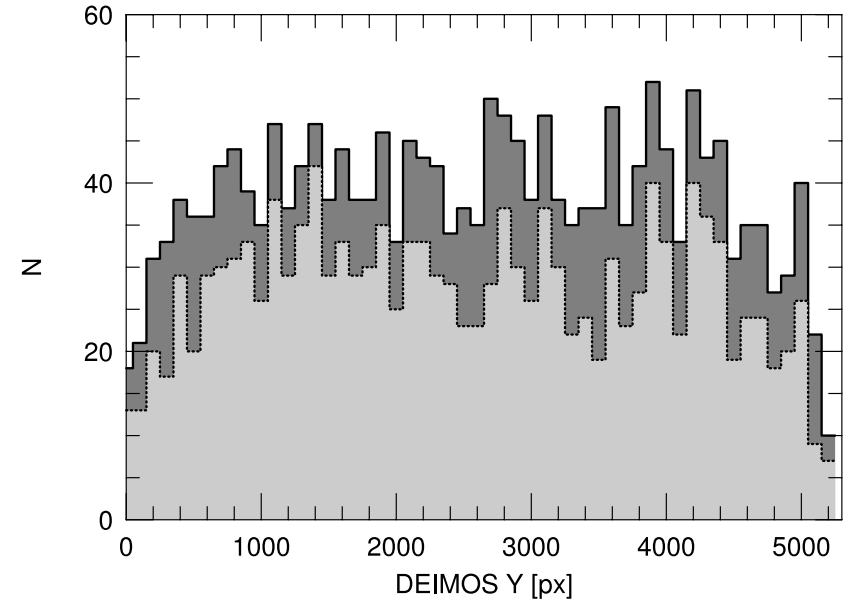

FIG. 5.- Number of targets placed on masks (dark grey histogram), and number of successful redshift measures (light grey), as a function of DEIMOS catalog $Y$ coordinate, which is parallel to the GOODS-N shorter axis. The fall-off at both edges is caused by the fact that galaxies and stars in these positions have a smaller probability of being placed on masks, since they are only considered as potential mask candidates a smaller number of times.

scopically. Our slitmasks targeted $70-80 \%$ of the available objects over the range $20.5<R<24$ that contains the majority of the galaxies, falling significantly below this level at the bright end (where sources were scarce) and in the faintest bin (containing faint targets against which selection was weighted during the slitmask design process). Panel (c) displays the fraction of these spectra which yielded secure redshifts (cf. Fig. 4 in Cowie et al. $(2004))$. Our success rate is high $(>90 \%)$ for galaxies brighter than $R=23.0$ and quickly decreases to below $60 \%$ at the faint limit. Panel (d) gives the fraction of all targets in the sample for which we obtained secure redshifts, and can be compared to the corresponding figures in Cohen et al. (2000) and Cowie et al. (2004). Overall, our survey obtained secure redshifts for $53 \%$ of all targets brighter than $R=24.4$ in the GOODS-N field. 


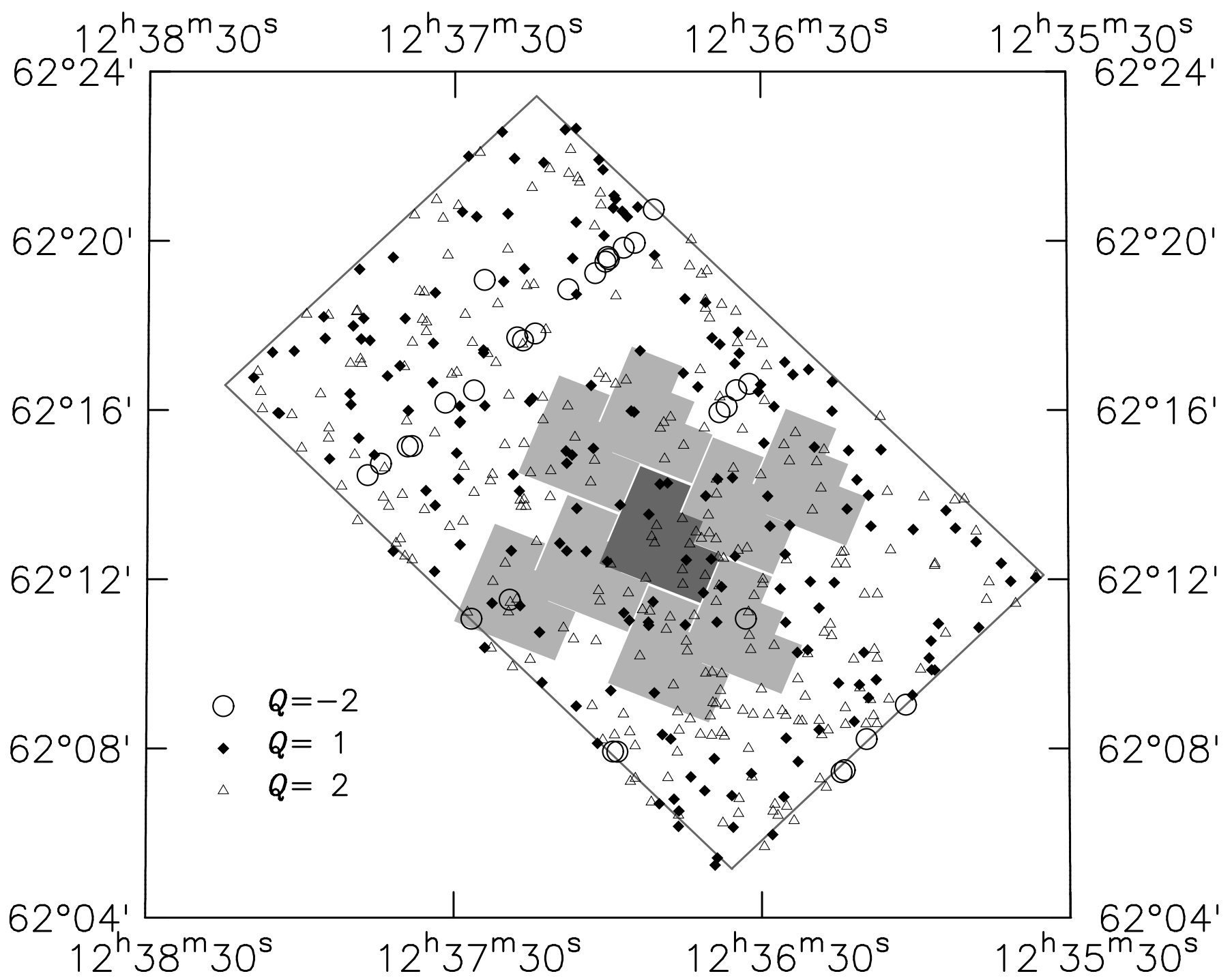

FIG. 6.- Projected sky distribution of objects for which no successful redshifts were measured. Plotting symbols distinguish objects for which no spectra were obtained because they fell on bad columns, gaps between CCDs, or at the edges of masks $(Q=-2)$, objects for which a spectrum was obtained, but no redshift could be deduced $(Q=1)$, and objects which may yet produce redshifts or for which only one spectral feature was identified on the spectra $(Q=2)$. Shaded regions indicate the HDF and flanking fields as in Fig. 3

Color effects. - The recent release of multicolor photometry for the GOODS-N field (Capak et al. 2004; Giavalisco et al. 2004) enables tests of color dependence in our sample. In the following analyses, we have matched our target catalog derived from the DEIMOS photometry with the Giavalisco et al. multicolor photometry derived from the v1.0 GOODS-N ACS images. Objects in both catalogs number 2816, with a detection rate of $2816 / 2911=90 \%$. Most non-matching galaxies in the DEIMOS catalog are found in regions just outside that covered by the v1.0 GOODS-N catalog. Figure 8 displays the distribution of the $V_{606}-i_{775}$ colors measured within a circular aperture of radius $3^{\prime \prime}$ for all galaxies placed on mask, galaxies with secure redshifts $(Q \geq 3)$ and galaxies with unmeasurable or insecure redshifts $(Q=1,2)$. The objects with failed redshift measurements are preferentially bluer than the successful targets. Figure 9 compares the colors of targets with successful and failed redshift measurements in different apparent magnitude ranges, and shows that the distribution of failed measurements changes from relatively flat to become bluer than the overall distribution near the faint limit.

The apparent color-magnitude distribution of the sample in common with the GOODS-N V1.0 catalogue is shown in Fig. 10] where panel (a) shows the entire sample (stars and galaxies). Panel (b) shows the subsample of objects that were placed on slits, while panel (c) discriminates targets in various redshift quality classes. Failed reshifts $(Q=1)$ are predominantly found at the faintest and bluest limits of the sample. A similar analysis made for the DEEP2 survey (Willmer et al. 2004), shows that most of these failed redshifts are likely to be of galaxies at high redshift, for which the [O II] lines fall outside the wavelength range of the DEIMOS setup used in the TKRS.

The sampling rate as a function of apparent $V_{606}-i_{775}$ color and magnitude is shown in Fig. 11] The sampling rate was measured in bins of 0.25 mag both in magnitude and color, and the shading in the figure represents 
TABle 3. TKRS Completeness

\begin{tabular}{lrrrrrrr}
\hline \hline$R_{A B}$ Range $^{\mathrm{a}}$ & $N_{\text {tot }} \mathrm{b}$ & $N_{\text {obs }}{ }^{\mathrm{c}}$ & $N_{z}^{\mathrm{d}}$ & $\begin{array}{c}F_{o b s} \mathrm{e} \\
(\%)\end{array}$ & $\begin{array}{c}F_{z}^{\mathrm{f}} \\
(\%)\end{array}$ & $\begin{array}{c}F_{z, \text { all }}{ }^{\mathrm{f}} \\
(\%)\end{array}$ & $\begin{array}{c}F_{z, \text { cum }} \mathrm{g} \\
(\%)\end{array}$ \\
\hline $17.0-17.5$ & 5 & 0 & 0 & 0 & 0 & 0 & 0 \\
$17.5-18.0$ & 17 & 0 & 0 & 0 & 0 & 0 & 0 \\
$18.0-18.5$ & 7 & 0 & 0 & 0 & 0 & 0 & 0 \\
$18.5-19.0$ & 10 & 2 & 2 & 20 & 100 & 20 & 5 \\
$19.0-19.5$ & 21 & 12 & 12 & 57 & 100 & 57 & 23 \\
$19.5-20.0$ & 33 & 14 & 14 & 42 & 100 & 42 & 30 \\
$20.0-20.5$ & 29 & 16 & 16 & 55 & 100 & 55 & 36 \\
$20.5-21.0$ & 57 & 44 & 43 & 77 & 98 & 75 & 49 \\
$21.0-21.5$ & 111 & 80 & 74 & 72 & 93 & 67 & 56 \\
$21.5-22.0$ & 152 & 119 & 112 & 78 & 94 & 74 & 62 \\
$22.0-22.5$ & 231 & 169 & 156 & 73 & 92 & 68 & 64 \\
$22.5-23.0$ & 330 & 248 & 222 & 75 & 90 & 67 & 65 \\
$23.0-23.5$ & 466 & 345 & 273 & 74 & 79 & 59 & 63 \\
$23.5-24.0$ & 671 & 500 & 353 & 75 & 71 & 53 & 60 \\
$24.0-24.4$ & 775 & 438 & 259 & 57 & 59 & 33 & 53 \\
& & & & & & & \\
\hline
\end{tabular}

a Apparent magnitude range.

${ }^{\mathrm{b}}$ Number of cataloged objects in magnitude range.

${ }^{\mathrm{c}}$ Number of spectroscopic targets in magnitude range.

d Number of secure galaxy redshifts measured in magnitude range.

e Differential fraction of objects targeted with slits.

${ }^{\mathrm{f}}$ Differential fraction of observed objects yielding secure redshifts.

${ }^{\mathrm{f}}$ Differential fraction of all objects yielding secure redshifts.

${ }^{\mathrm{g} C u m u l a t i v e}$ fraction of all objects yielding secure redshifts.

different sampling rates as described in the key. Panel (a) shows the ratio between number of objects placed on masks, relative to the number of objects within the same color-magnitude limits in the sample. The sampling rate of the TKRS is higher than $65 \%$ for the majority of the color-magnitude bins, though this rate falls for fainter and bluer galaxies. Panel (b) shows the success rate; i.e., the number of spectra that produced redshifts compared to the total placed on masks. The completeness level is higher than $80 \%$ to about $R \lesssim 23$, beyond which it falls due to the lower $S / N$ of absorption line spectra, and the loss of key spectral features outside the wavelength range of the spectra.

Surface brightness effects. - Figure [12 shows isophotal area plotted against $z$ magnitude for all of the spectroscopic targets in our survey. Both photometric parameters are from the HST ACS imaging catalog of Giavalisco et al. (2004), with the magnitudes measured in the outermost aperture $\left(3^{\prime \prime}\right)$. In this plot we again distinguish objects in the various redshift quality classes of our survey. The stellar and galaxy sequences are well differentiated to the limiting $z$ magnitude. Objects with uncertain redshifts include faint stars as well as galaxies of varying brightness. Many of the brighter objects with uncertain redshifts $(Q=2)$ failed because of poorly defined extraction windows when creating the 1-D spectra. This problem will be revisited in a later re-analysis of the 2-D spectra and a corrected version of the cata$\log$ will be issued. Failed redshifts $(Q=1)$, as opposed to uncertain ones, start to occur at $z \approx 22$. However, the diagram provides no strong evidence that the $Q=1$ galaxies have lower surface brightnesses than the subsample with secure redshifts.

\subsection{Comparison with other surveys}

Areas within the GOODS-N field have already been the subject of intensive study by deep surveys of the HDF-N region that lies within it. As mentioned in 11 several research teams have obtained redshifts for galaxies in this part of the sky, among them Cohen et al. (1996); Cowie et al. (1996); Steidel et al. (1996); Lowenthal et al. (1997); Phillips et al. (1997); Moustakas et al. (1997); Cohen et al. (2000); Cohen (2001); Dawson et al. (2001). Recent work by other observing teams using Keck II with DEIMOS to survey the GOODS-N field include the companion survey of Cowie et al. (2004) and the higher-resolution survey of red galaxies by Treu et al. (2004). We can compare our measurements with the findings of these independent surveys to gauge the accuracy of our redshifts and our quality assessments.

The results of the redshift comparisons are depicted graphically in Fig. 13] and key results are summarized in Table 4 For each survey, the table provides the number of objects in common with the TKRS sample in addition to robust measures of both the difference in measured redshift and the dispersion in that difference. For purposes of this discussion we defined two redshift measurements to "agree" if they differ by less than $|\Delta z| \leq 0.002$ $\left(|\Delta v| \lesssim 600 \mathrm{~km} \mathrm{~s}^{-1}\right)$; this excludes galaxies with severely discrepant redshifts and does not significantly bias the resulting measures of redshift offset and dispersion. To quantify the redshift differences with our work we computed the offsets via the biweight, while the median absolute deviation from the median (MAD, with multiplicative scaling factor 9) served as a robust estimator of the dispersion in the offsets (Beers et al. 1990). We now discuss the results of the comparison for individual surveys.

In an LRIS survey covering a region centered on the HDF and Flanking Fields, Cohen et al. obtained spectra for most galaxies with $R \leq 23.5$ in the Flanking Fields and $R \leq 24.5$ in the HDF-N (Cohen et al. 1996, 

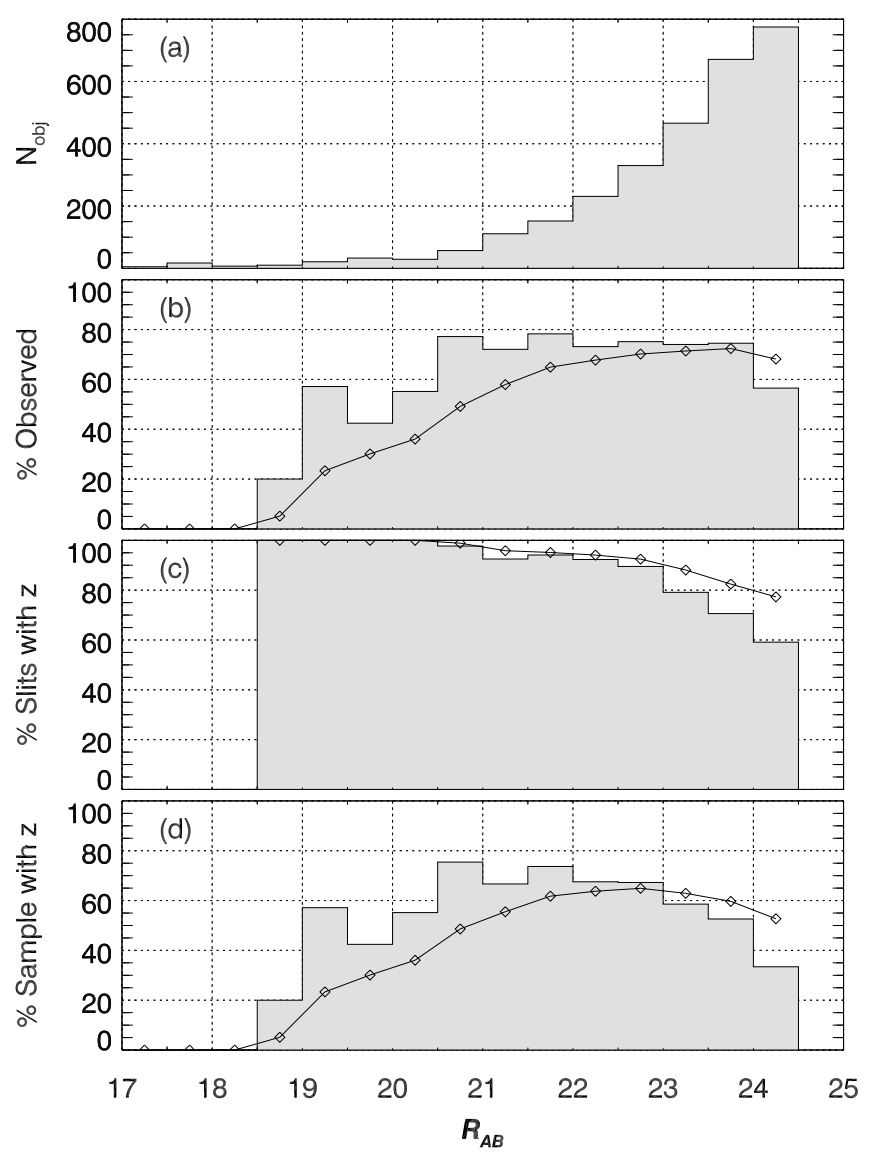

FIG. 7.- Completeness indicators as a function of $R_{A B}$ magnitude in the TKRS sample. (a) The number of available targets $N_{o b j}$ per $0.5 \mathrm{mag}$ bin in our GOODS-N field target catalog, to the limiting magnitude of $R \leq 24.4$. (b) Differential fraction of available targets (relative to the total sample available within each $0.5 \mathrm{mag}$ bin) for which spectra were acquired (shaded histogram), or the integrated fraction to that magnitude bin (connected diamonds). (c) Differential and integrated fraction of spectra which yielded secure redshifts. (d) Differential and integrated fraction of available targets which yielded secure redshifts, including objects not observed.

Table 4. Comparison of TKRS with Other Surveys

\begin{tabular}{lcrrr}
\hline \hline \multicolumn{1}{c}{ Survey } & Ref. & $N_{o b j}{ }^{\mathrm{a}}$ & $\begin{array}{r}\Delta v_{B I} \mathrm{~b}^{\mathrm{b}} \\
\left(\mathrm{km} \mathrm{s}^{-1}\right)\end{array}$ & $\begin{array}{r}S_{M A D}(\Delta v)^{\mathrm{c}} \\
\left(\mathrm{km} \mathrm{s}^{-1}\right)\end{array}$ \\
\hline Cohen et al. HDF-N & 1,2 & 254 & 52.9 & 174.3 \\
DEEP1 HDF-N & 3,4 & 60 & 27.2 & 99.8 \\
Cowie et al. GOODS-N & 5 & 378 & -15.5 & 140.5 \\
Treu et al. GOODS-N & 6 & 131 & -14.3 & 42.2 \\
\hline
\end{tabular}

References. - (1) Cohen et al. (2000); (2) Cohen (2001); (3) Lowenthal et al. (1997); (4) Phillips et al. (1997); (5) Cowie et al. (2004); (6) Treu et al. (2004).

${ }^{a}$ Number of objects with secure redshifts in both surveys.

${ }^{\mathrm{b}}$ Difference in radial velocity (in the sense $v-v_{T K R S}$ ) among secure redshifts, as quantified by the Biweight.

${ }^{c}$ Robust estimate of dispersion in radial velocity differences as quantified by the median absolute deviation from the median, scaled by an appropriate factor (Beers et al. 1990).

2000; Cohen 2001). The redshift catalogue of these works also contains measurements obtained from the literature; however, in the present comparison we concentrate on the uniform sample of 413 galaxies measured by J. Cohen

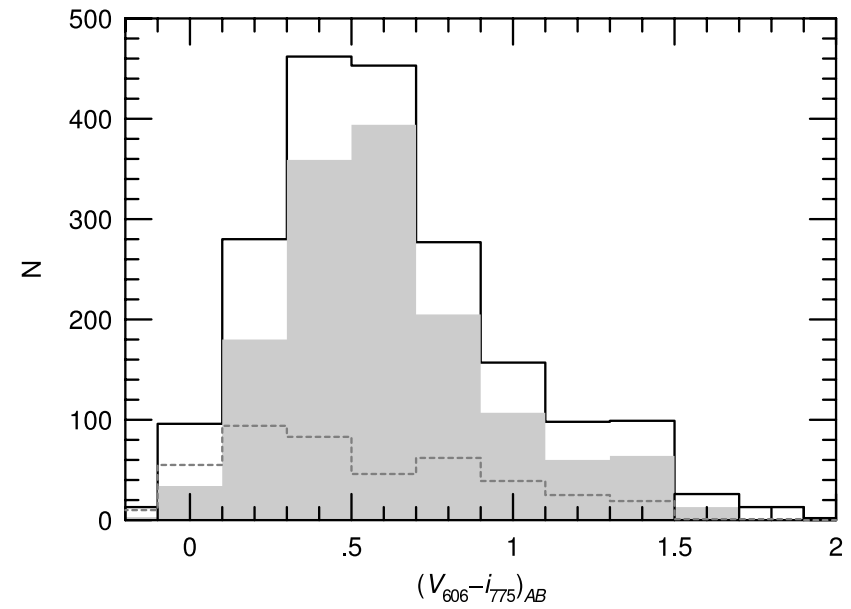

FIG. 8.- Number of galaxies placed on masks (solid black line), and number of redshifts measured (grey histogram), as a function of the $V_{606}-i_{775}$ color. Also shown is the distribution of galaxies with redshift qualities $Q=1$ and 2 (dashed line). For the bluest galaxies the success rate (ratio of grey histogram to solid line) is under $50 \%$, and, as discussed in the text and Fig. 10 is likely due to unidentified high-redshift galaxies.

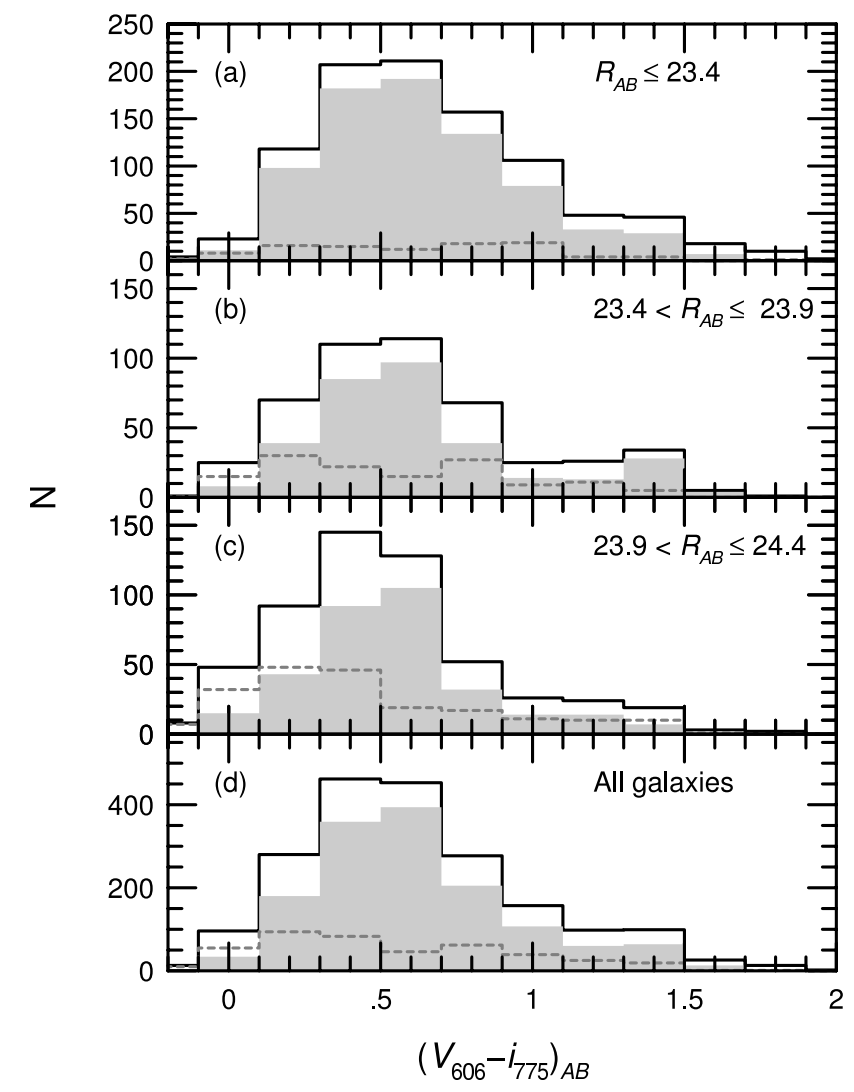

FIG. 9.- Histogram showing the number of galaxies placed on masks (solid black line) and number of redshifts measured (grey histogram), as a function of the $V_{606}-i_{775}$ color. Also shown is the distribution of failed redshift measurements (dashed line). Panels (a) through (c) show the color distributions for different magnitude ranges, and panel (d) shows the distribution for the entire sample. While the color distributions of galaxies placed on masks and of those successfully observed do not change significantly with magnitude, the color distribution of redshift failures shows a clear trend of becoming bluer at fainter apparent magnitude. 


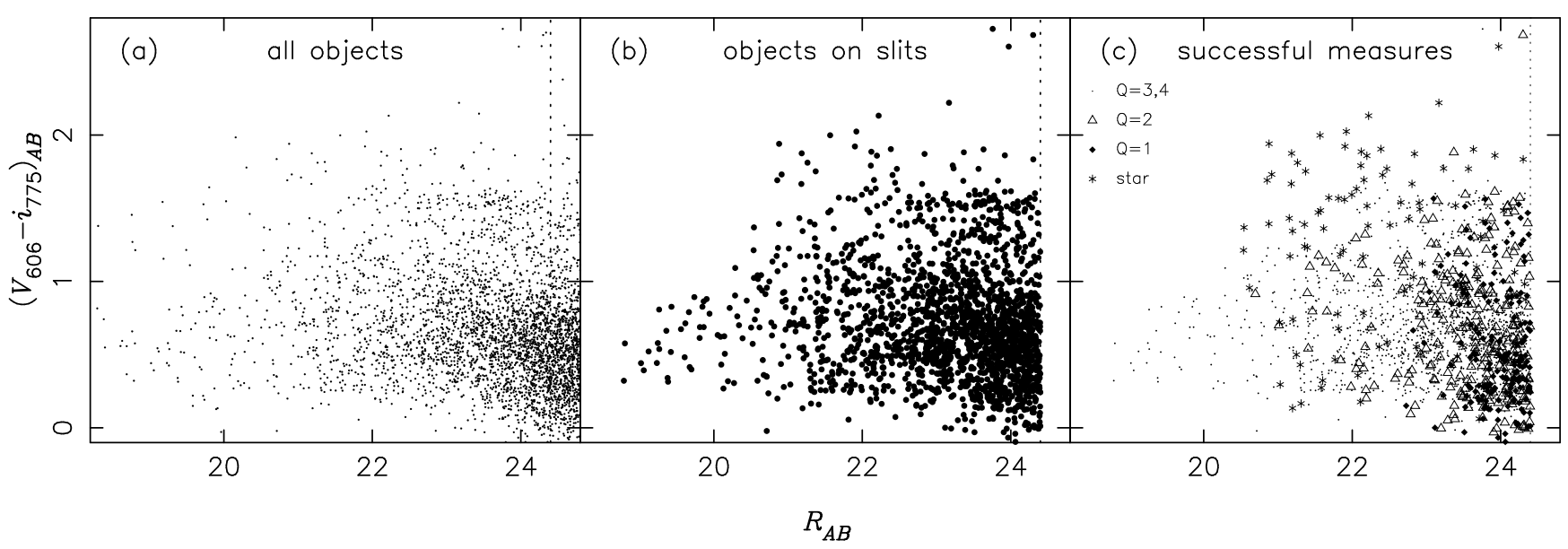

FIG. 10.- Apparent color-magnitude diagram for objects placed on TKRS masks. (a) The total sample of stars and galaxies in catalog; the dotted line represents the nominal limit of $R_{A B}=24.4$ used in the selection of the spectroscopic sample. (b) The distribution of objects placed on masks. (c) Observed targets, including galaxies with secure redshifts $(Q=3,4)$, dubious measures $(Q=2)$, objects for which no redshift can be measured $(Q=1)$, and confirmed stars $(Q=-1)$. The $Q=1$ sample generally consists of blue and faint objects, suggesting that these are likely to be high-redshift galaxies for which the [O II] $\lambda \lambda 3726,3729$ lines fall outside the TKRS spectral window.

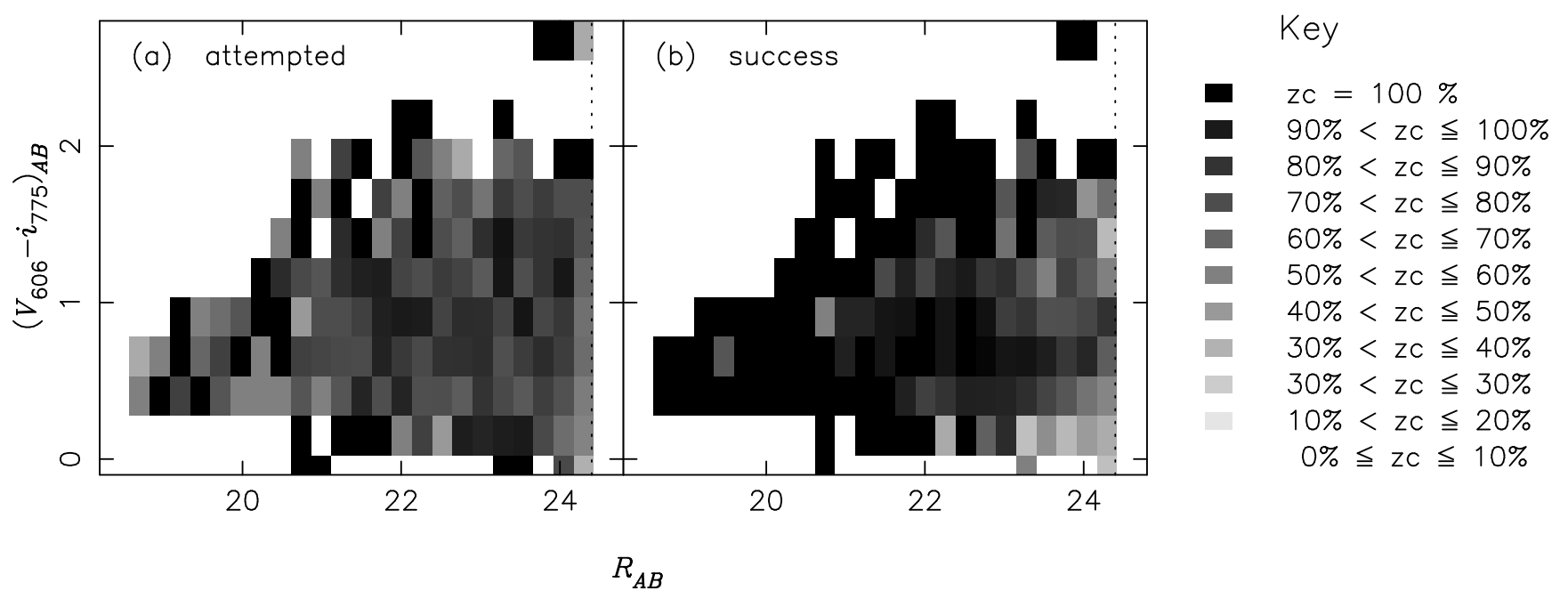

FIG. 11. - Sampling rate for the TKRS sample. (a) Sampling rate (ratio of number of galaxies + stars observed to the number available within the bin) vs. $H S T V_{606}-i_{775}$ color and magnitude, within $0.25 \mathrm{mag}$ bins. (b) Success rate (ratio between the number of successful measurements and the number placed on masks) vs. HST $V_{606}-i_{775}$ color and magnitude. The legend to the right of the figure explains the color coding.

using Keck I with LRIS. For 254 galaxies with redshift in rough agreement with ours (Fig. 13(a)) the biweight difference is $z_{\text {Cohen }}-z_{T K R S}=53 \mathrm{~km} \mathrm{~s}^{-1}$ with a dispersion of $174 \mathrm{~km} \mathrm{~s}^{-1}$. Cohen et al. (2000) obtained secure redshifts for 52 targets measured poorly in the TKRS sample $(Q=1$ or 2$)$.

Another LRIS sample in the HDF-N and Flanking Fields is that observed by the DEEP1 survey team, who targeted primarily $U$ - and $B$-band dropouts (Lowenthal et al. 1997) and compact galaxies
(Phillips et al. 1997). Of 114 galaxies in common, 60 have redshifts within $|\Delta z|<0.002$ of the TKRS measurement, and the distribution of redshift differences is shown in Fig. 13 (b). The offset in redshift is $z_{D E E P}-z_{T K R S}=$ $27 \mathrm{~km} \mathrm{~s}^{-1}$ with a dispersion of $100 \mathrm{~km} \mathrm{~s}^{-1}$. The DEEP1 sample provides 7 reliable measurements of galaxy redshifts for which the TKRS redshift has a quality of 1 or 2 .

The Cowie et al. (2004) sample, which used the same instrument, configuration, integration time, and survey 


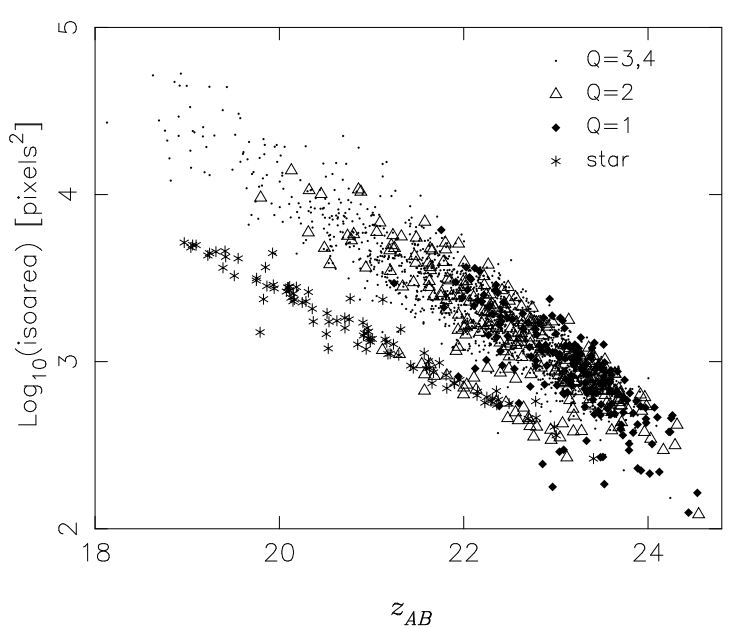

FIG. 12.- Apparent object size (ACS isophotal image area) vs. apparent $z$ magnitude measured in the outermost aperture $\left(3^{\prime \prime}\right)$ on ACS images (Giavalisco et al. 2004) for the 1981 sources observed in our redshift survey. Different symbols distinguish objects with secure redshifts $(Q=3,4)$, uncertain redshifts $(Q=2)$, no measured redshifts $(Q=1)$, and stars $(Q=-1)$.

region as the TKRS sample, features 830 galaxies in common with TKRS, of which 452 have secure redshifts in both works; see Fig. 13(c). Among the 378 redshifts in agreement, the offset is $z_{\text {Cowie }}-z_{T K R S}=-16 \mathrm{~km} \mathrm{~s}^{-1}$ with a dispersion of $141 \mathrm{~km} \mathrm{~s}^{-1}$. A total of 56 galaxies for which TKRS was unable to obtain a redshift (i.e., $Q=1,2$ ) were successfully measured by Cowie et al. Conversely, TKRS was able to measure redshifts for 50 galaxies that were observed by Cowie et al. but for which they were unable to get a reliable redshift.

Finally, in Fig. 131(d) we compare our redshifts with measurements obtained by Treu et al. (2004), who targeted preferentially red galaxies in the GOODS-N field using Keck II with DEIMOS. Their higher-resolution survey used the $1200 \mathrm{l} \mathrm{mm}^{-1}$ grating to acquire exposures ranging from $5-10 \mathrm{~h}$, as compared to $1 \mathrm{~h}$ for TKRS. Of 199 galaxies in common with our catalog, 178 have secure redshifts in the Treu et al. sample and 131 have secure redshifts in both samples; only one redshift measurement is significantly discrepant $(|\Delta z| \geq 0.0025)$. Among 130 redshifts which agree the offset between these samples is $-14 \mathrm{~km} \mathrm{~s}^{-1}$ with a dispersion of $42 \mathrm{~km} \mathrm{~s}^{-1}$, so that this sample represents the lowest dispersion with respect to our redshifts. These measurements from Treu et al. also provide an estimate on how the increase of exposure time affects the redshift success rate. Of the 18 objects in common whose redshifts in the TKRS were uncertain $(Q=2)$ or could not be measured, $7(39 \%)$ agree with the measurements with longer exposures. This number corresponds to $\sim 4 \%(7 / 179)$ of galaxies within the sample in common. An additional 6\% (11/179) of objects yielded successful redshift measurements with the longer integration times.

\subsection{Accuracy of redshift measurements}

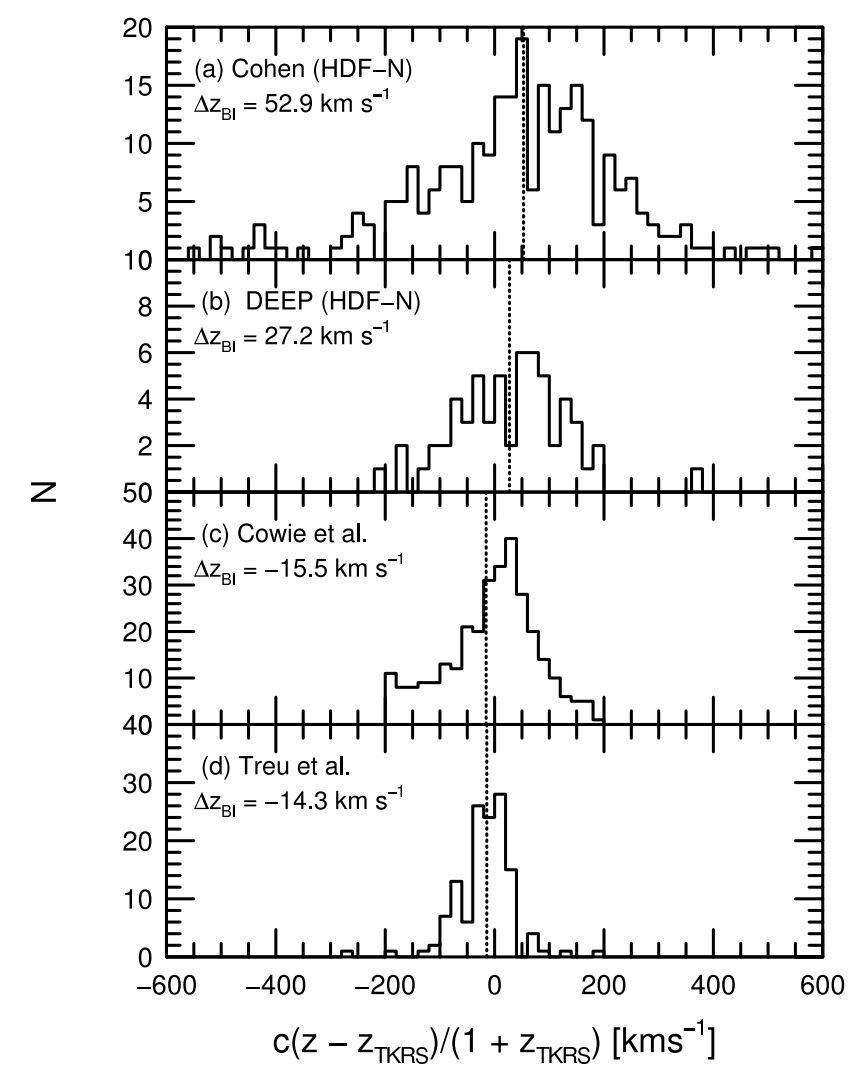

FIG. 13.- Histogram showing the redshift differences between galaxies with secure measurements in the TKRS vs. four other extensive samples in the GOODS-N region. Each panel shows the bi-weight location (dotted line) and its value (Beers et al. 1990). (a) Redshift differences for galaxies with secure measurements from LRIS in Cohen et al. (2000) and Cohen (2001). (b) Redshift differences between TKRS and secure measurements of Phillips et al. (1997) and Lowenthal et al. (1997), also using LRIS. (c) Redshift differences with the Cowie et al. (2004) sample of galaxies, which used similar instrumental setup and integration times as the TKRS. (d) Redshift differences with the Treu et al. (2004) sample of galaxies observed with DEIMOS but using higher resolution and longer integration times than this work.

The independent redshift measurements available for a significant number of galaxies in our catalog allow us to estimate the uncertainty in our redshifts. Of the four comparable catalogs described in 4.3 the most useful ones for comparison are the DEIMOS surveys of Treu et al. and Cowie et al., since they use the same instrument as our survey. Of these two, the Treu et al. sample is expected to be more accurate by virtue of its higher dispersion (twice that of TKRS) and longer exposure time (typically 5 times longer). The dispersion in redshift measurements between these two surveys should thus be dominated by errors in the TKRS sample. As shown in the last column of Table 4 the robust estimate of dispersion in the redshift differences of these surveys is $42 \mathrm{~km} \mathrm{~s}^{-1}$. Assuming that the TKRS velocity uncertainties are at least a factor of two larger than those of Treu et al. due to the difference in dispersion, we estimate an average uncertainty of about $38 \mathrm{~km} \mathrm{~s}^{-1}$ for TKRS, with the caveat that the sample of galaxies used in the comparison consists almost exclusively of early-type galaxies and is thus not representative of the GOODS-N region 
as a whole.

The most comparable of the existing surveys to TKRS is the Cowie et al. sample made at the same dispersion and wavelength interval with DEIMOS. The dispersion in redshift measurements for the sample of 363 common targets between these two surveys is $141 \mathrm{~km} \mathrm{~s}^{-1}$. Assuming the two surveys have comparable uncertainties, this implies an error of $99 \mathrm{~km} \mathrm{~s}^{-1}$ for each. This value is over a factor of two higher than that measured compared to the Treu et al. sample. This may be due to Cowie et al. surveying fainter galaxies over a wider range of spectral types than Treu et al., as well as different slit orientations used by Cowie et al. vs. TKRS. Being conservative, we conclude that the characteristic uncertainty in our redshift measurements is $\sigma \approx 100 \mathrm{~km} \mathrm{~s}^{-1}$ or better.

\subsection{Accuracy of Quality Codes}

The existence of independent redshift measurements for many objects in our catalog allows us to assess the accuracy of our redshift quality categories. In other words, are the quality code 3 redshifts (which by definition should be correct at the $95 \%$ confidence level) truly correct $95 \%$ of the time? In Table [2] we match the TKRS catalogue with a list of redshifts measured by other authors. This list collated measurements from Cohen et al. (1996); Cowie et al. (1996); Lowenthal et al. (1997); Phillips et al. (1997); Moustakas et al. (1997); Cohen et al. (2000); Cohen (2001); Dawson et al. (2001); Steidel et al. (2003) and Cowie et al. (2004). The collated catalog was then correlated against the DEIMOS catalog, providing a list of matching objects. In assessing the number of correct redshift identifications, we assume that all discrepant redshifts result from errors in the present sample, thus obtaining a firm lower limit on the quality of our measurements. The final column in this table shows that the $Q=4$ redshifts agree with previous measurements in $94 \%$ of cases, establishing this as the lower limit to the accuracy of our most secure redshifts. For $Q=3$, the corresponding lower limit on the success probability is $91.6 \%$. If we make the more reasonable assumption that the mis-identifications are equally split between TKRS and other samples, the success rate increases to $96 \%(Q=3)$ and $97 \%(Q=4)$. We conclude that the respective intended confidence levels of $95 \%$ and $99 \%$ for the category 3 and 4 redshift measurements are reasonably accurate.

\subsection{Comparison with photometric redshifts}

We have compared the spectroscopic redshifts with the photometric redshift catalog of Capak et al. (2004). These redshift estimates are based on the ultradeep imaging in eight colors from the $U$ to the near IR which is described in Capak et al. (2003) and are computed using the Bayesian code of Benitez (2000). This assigns odds that the redshift lies close to the estimated value and we have only used those cases where these odds lie above 90\%. The imaging data also saturate at bright magnitudes so we have restricted the comparison to sources with $R>20$. This gives a sample of 1078 sources with both spectroscopic and photometric redshifts which we compare in Fig. 14. Of the sources, 8 are broad line AGN where the photometric estimates may be poorer since the templates do not properly model such objects. We have

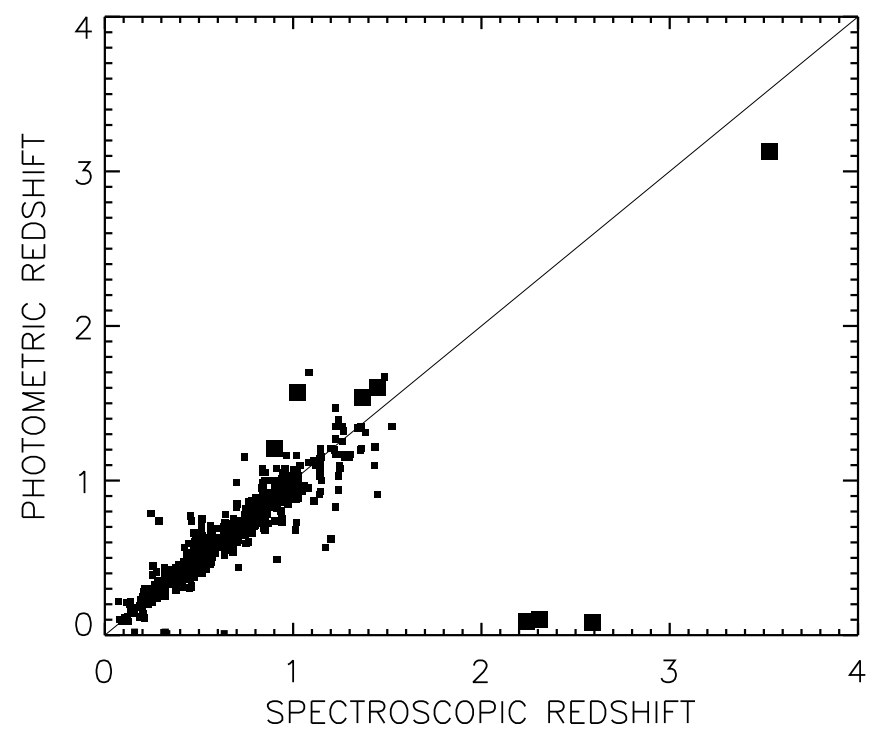

FIG. 14. - Photometric redshift versus spectroscopic redshift for the 1078 sources with $R<24$ where both have been measured (solid squares). Objects with broad-line AGN spectra are distinguished with a larger symbol. Only 38 of the photometric redshifts differ by more than $30 \%$ from the spectroscopic redshift. Five of these cases are broad-line AGN.

distinguished these objects with larger symbols. Nearly all $(97 \%)$ of the photometric redshifts agree to within $30 \%$ with the spectroscopic redshift, consistent with the expectation from the probabilities assigned to the photometric redshifts. This agreement is independent of magnitude over the $20<R<24$ range.

\section{ANALYSIS}

\subsection{Redshift distributions}

The distribution of galaxies in our survey as a function of $R_{A B}$ and redshift appears in Fig. [15] Also shown in this plot and in Table 5 is the median redshift as a function of magnitude. The plot compares these values to the median redshifts in the Cohen et al. (2000) survey; both samples are uncorrected for incompleteness. For bins brighter than $R=22.5$, our median redshifts are significantly lower than those of Cohen et al.. We note that the present survey has a cumulative completeness of just over $60 \%$ at $R=22.5$, compared to $>90 \%$ for Cohen et al.. In the faintest magnitude bin of the Cohen et al. HDF sample (23.5-24.0) the differential completeness drops to the same $50 \%$ value as our survey, and as a result the median redshifts in this magnitude range $\left(z_{M}=0.79\right.$ for Cohen et al. vs. 0.80 in our sample) are consistent.

Figure [16] shows the redshift distribution of galaxies with secure measurements. This plot shows the number of galaxies contained within a velocity window of width $\pm 750 \mathrm{~km} \mathrm{~s}^{-1}$ at any given redshift and thus isolates concentrations of galaxies in redshift space. The window width of $1500 \mathrm{~km} \mathrm{~s}^{-1}$ is well-matched to the numerous structures with velocity dispersions of $\sigma=500$ $700 \mathrm{~km} \mathrm{~s}^{-1}$ reported by Cohen et al. (2000) in the HDF. Numerous concentrations of galaxies are seen in the sample, most notably those at $z=0.51,0.56,0.64,0.85$, and 1.02. These structures are equally prominent in the pie diagrams shown in Fig. 17(a) and (b) which plot the projected linear offset from the field center (along the $\alpha$ and 


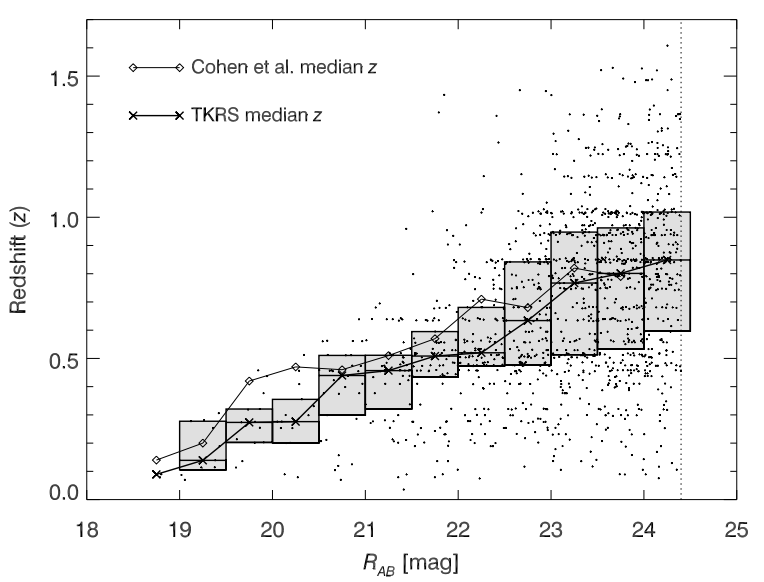

FIG. 15. - Redshift vs. $R$ magnitude for targets with secure redshifts. Small pluses indicate individual objects in the TKRS survey. Boxes indicate the interquartile range of redshifts within respective $0.5 \mathrm{mag}$ bins; each has a horizontal line and $\times$ which indicate the median redshift within that bin. The median redshifts of Cohen et al. (2000) are shown for comparison. The vertical dotted line at right represents the apparent magnitude limit of the present survey at $R_{A B}=24.4$.

Table 5. Median Redshift in Bins of $R$

\begin{tabular}{crccc}
\hline \hline$R_{A B}$ Range $^{\mathrm{a}}$ & $N_{\text {obj }}{ }^{\mathrm{b}}$ & $\mathrm{Q}^{\mathrm{c}}$ & Median $^{\mathrm{d}}$ & $\mathrm{Q3}^{\mathrm{e}}$ \\
\hline $18.5-19.0$ & 2 & $\ldots$ & 0.09 & $\ldots$ \\
$19.0-19.5$ & 11 & 0.11 & 0.14 & 0.28 \\
$19.5-20.0$ & 13 & 0.20 & 0.27 & 0.32 \\
$20.0-20.5$ & 15 & 0.20 & 0.28 & 0.36 \\
$20.5-21.0$ & 33 & 0.30 & 0.44 & 0.51 \\
$21.0-21.5$ & 57 & 0.32 & 0.46 & 0.51 \\
$21.5-22.0$ & 93 & 0.43 & 0.51 & 0.60 \\
$22.0-22.5$ & 133 & 0.47 & 0.52 & 0.68 \\
$22.5-23.0$ & 193 & 0.48 & 0.63 & 0.84 \\
$23.0-23.5$ & 254 & 0.51 & 0.77 & 0.95 \\
$23.5-24.0$ & 335 & 0.53 & 0.80 & 0.96 \\
$24.0-24.4$ & 248 & 0.60 & 0.85 & 1.02 \\
& & & & \\
\hline
\end{tabular}

apparent magnitude range.

${ }^{b}$ Number of secure TKRS redshifts in magnitude range.

${ }^{\mathrm{c}}$ Lower quartile redshift within magnitude range.

${ }^{\mathrm{d}}$ Median redshift within magnitude range.

e Upper quartile redshift within magnitude range.

$\delta$ axes, respectively) vs. redshift. These figures illustrate that some of the structures span the GOODS-N field, while others (particularly those at $z=0.85$ and 1.02 ) are spatially clumped.

\subsection{Color distribution}

In Fig. 18 we show the rest-frame color-magnitude diagram based on the HST ACS photometry for all galaxies with secure TKRS redshifts appearing in the Giavalisco et al. (2004) catalog. The rest-frame parameters were calculated by convolving the CCD and filter throughputs of ACS with spectral energy distributions of galaxies in the Kinnev et al. (1996) atlas, a more detailed description of which will be presented elsewhere (Willmer et al. 2004). As seen in Fig. 18(b), the restframe galaxy colors are bi-modal about a minimum near $(U-B)_{0}(A B) \approx 0.9\left(\right.$ corresponding to about $(U-B)_{0} \approx$
0.3 in the Vega system). This effect has also been noted in the DEEP1 survey (Im et al. 2002; Weiner et al. 2004) in the Groth Survey Strip (which also used HST photometry), as well as in the Sloan Digital Sky Survey (Strateva et al. 2001; Hogg et al. 2002) and COMBO-17 survey (Bell et al. 2003). Figure [18(a) illustrates that the the color bi-modality is clearly present to $z>1.1$, which is beyond the limits both of Bell et al. (2003) and Weiner et al. (2004). The lack of low-luminosity red galaxies, also noted by Weiner et al. (2004), is most likely to be a real effect.

\section{SUMMARY}

We have described our deep redshift survey of the GOODS-N region using DEIMOS, the result of which is a catalog of photometry, astrometry, redshifts and redshift quality assessments for 1440 confirmed galaxies/AGN and stars in the $10^{\prime} \times 16^{\prime}$ GOODS-N region. This sample is $53 \%$ complete to a limiting magnitude of $R_{A B}=24.4$ and has a median redshift of $z_{M}=0.65$. In conjunction with an accompanying DEIMOS survey by Cowie et al. (2004), it provides a substantial and homogeneous sample of new redshifts and spectra in this important area of the sky.

The TKRS catalog will continue to evolve as DEIMOS pipeline processing algorithms are refined, and we expect that a significant number of galaxies may be promoted from redshift quality class 2 (uncertain) to 3 (> $90 \%$ Secure). To ensure that researchers obtain access to the revised data, we have established a website ${ }^{10}$ to disseminate all data products resulting from this survey. In addition to our most recent catalog of DEIMOS photometric and spectroscopic measurements of all targets in the GOODS-N field, astronomers can currently obtain the wavelength-calibrated 1-D spectra and will soon be able to download the sky-subtracted 2-D images from which the 1-D spectra were extracted. These resources will allow others to measure velocity widths and spectral indices for the sample, promoting the study of fundamental galaxy properties at moderate redshift.

This project was the brainchild of David Koo, who conceived of the survey, formulated a detailed plan for executing it, and (with support from Sandra Faber) served as its principal advocate. The survey would not have been completed without the generosity of the DEEP2 Redshift Survey Team, which not only developed the sophisticated data reduction pipeline for DEIMOS, but also donated a significant amount of their DEIMOS observing time to complete the survey. We are indebted to Garth Illingworth and Pieter van Dokkum for acquiring several of the DEIMOS images required for the photometric survey. Additionally, we thank Drew Phillips for helping process the slitmask designs and for use of his cosmic ray rejection algorithm, Ben Weiner for useful discussions and for running his redshift measuring code independently on this sample, plus Tommaso Treu and Richard Ellis for sharing redshifts from their GOODS-N survey prior to publication.

The National Science Foundation supported our survey through grant AS-0331730. This research has made

\footnotetext{
10 http://www2.keck.hawaii.edu/science/tksurvey
} 


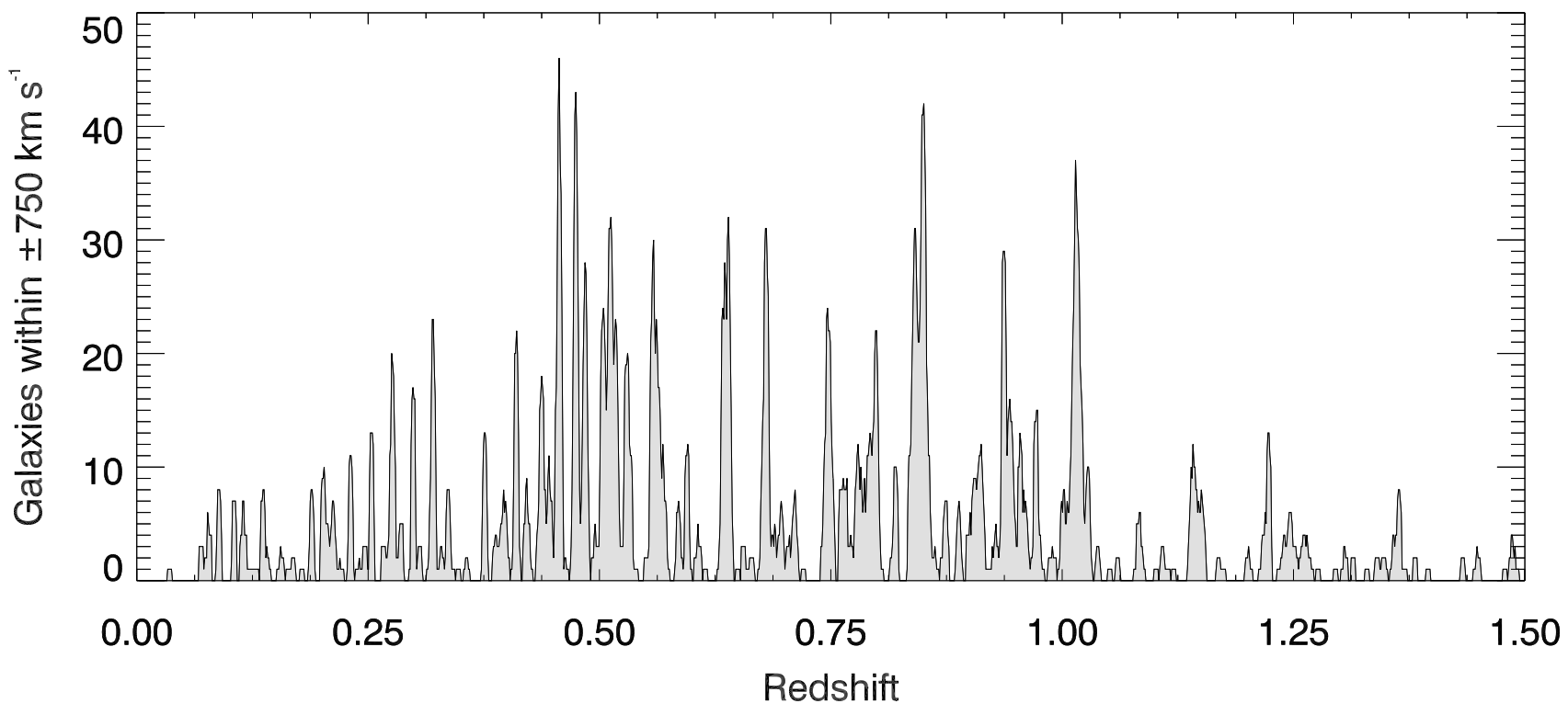

FIG. 16. - Redshift distribution of galaxies with secure measurements in the present sample. The raw marginal distribution was smoothed with a moving top hat kernel tuned to highlight structures similar in velocity dispersion to those noted in the HDF (Cohen et al. 2000). The ordinate represents the number of galaxies within $\pm 750 \mathrm{~km} \mathrm{~s}^{-1}$ at any given redshift. Several galaxies at high redshift are not shown.
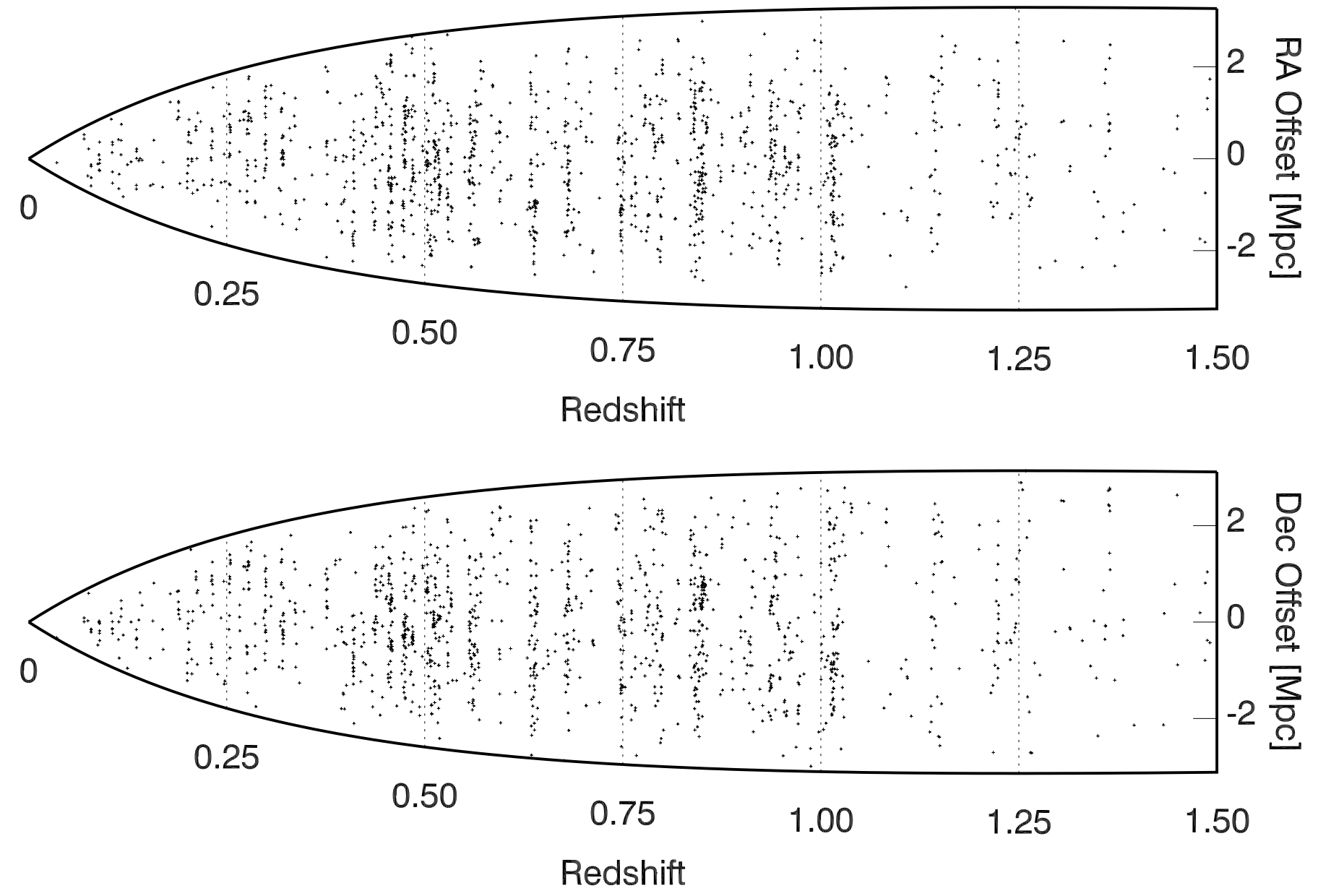

FIG. 17.- Pie diagrams showing the spatial distribution as a function of redshift for all galaxies with secure measurements in our survey. (a) Projected distance of each galaxy from the center of the GOODS-N field in the direction of Right Ascension vs. redshift. (b) Same, for Declination. In each plot, the outer envelope represents the linear separation corresponding to the 18.4 width of the field at that redshift. Note the numerous walls correponding to peaks in the marginal distribution of redshifts seen in Fig. [16] Dotted lines indicate lines of constant redshift. Cosmological parameters $h_{0}=0.75$ and $q_{0}=0.5$ are assumed in computing the spatial offsets. 


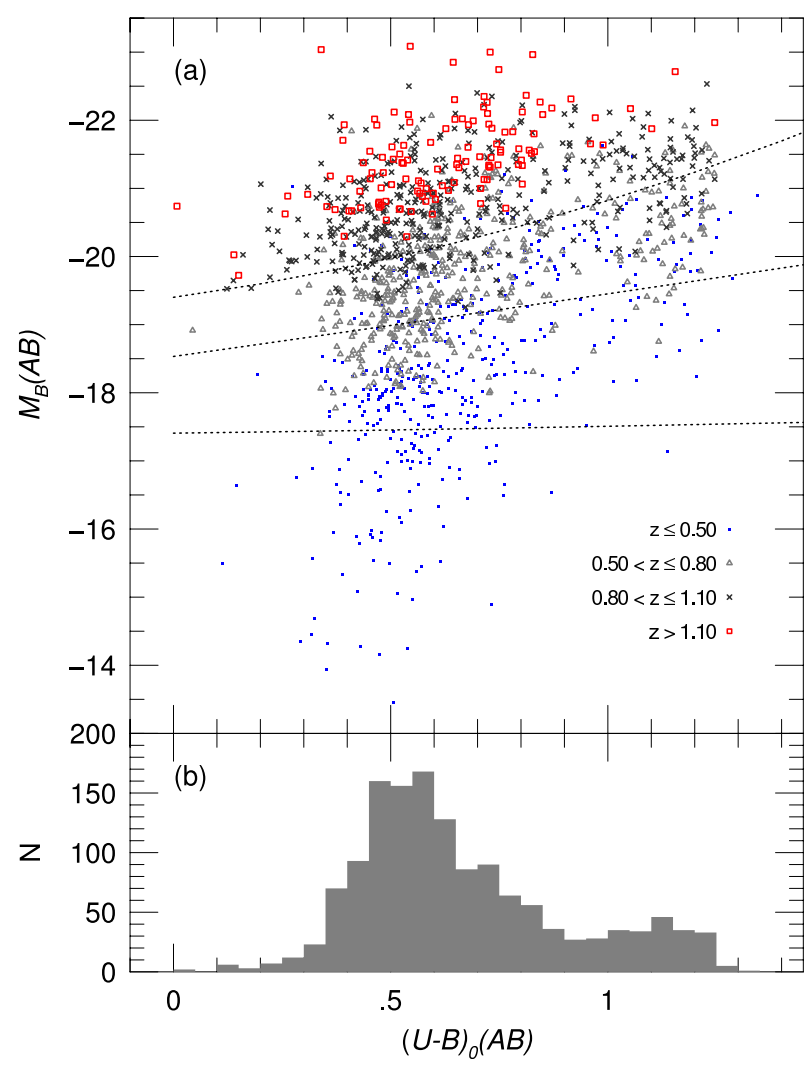

FIG. 18. - Rest-frame color-magnitude diagram and color distribution. (a) Rest-frame brightness vs. color for galaxies with TKRS redshifts in the Giavalisco et al. (2004) catalog. The three dashed lines show the limiting absolute magnitude as a function of restframe color at three different redshifts: $z=0.50$ (lower curve), $z=0.80$ (middle curve) and $z=1.10$ (top curve). The symbols are also coded by redshift interval as shown in the figure. (b) Distribution of $(U-B)_{0}$ colors in $0.05 \mathrm{mag}$ bins. The trough at $(U-B)_{0}(A B) \approx 0.9$ is consistent with that seen in other samples of galaxies. use of the NASA/IPAC Extragalactic Database (NED) which is operated by the Jet Propulsion Laboratory, California Institute of Technology, under contract with the National Aeronautics and Space Administration. This research also relied upon data from the Sloan Digital Sky Survey (SDSS). Funding for the creation and distribution of the SDSS Archive has been provided by the Alfred P. Sloan Foundation, the Participating Institutions, the National Aeronautics and Space Administration, the National Science Foundation, the U.S. Department of Energy, the Japanese Monbukagakusho, and the Max Planck Society. The SDSS is managed by the Astrophysical Research Consortium (ARC) for the Participating Institutions. The Participating Institutions are The University of Chicago, Fermilab, the Institute for Advanced Study, the Japan Participation Group, The Johns Hopkins University, Los Alamos National Laboratory, the Max-Planck-Institute for Astronomy (MPIA), the MaxPlanck-Institute for Astrophysics (MPA), New Mexico State University, University of Pittsburgh, Princeton University, the United States Naval Observatory, and the University of Washington. Our special thanks to Andy Connolly, who helped arrange our access to the SDSS data prior to the DR1 release.

We thank the anonymous reviewer for helpful suggestions which improved the quality of the manuscript. C. N. A. W. thanks the W. M. Keck Observatory for its hospitality throughout the visit during which much of this work was completed.

We also wish to recognize and acknowledge the highly significant cultural role and reverence that the summit of Mauna Kea has always had within the indigenous Hawaiian community. It is a privilege to be given the opportunity to conduct observations from this mountain.

\section{REFERENCES}

Bell, E. F., Wolf, C., Meisenheimer, K., Rix, H.-W., Borch, A., Dye, S., Kleinheinrich, M., \& McIntosh, D. H. 2004, ApJ, submitted, astro-ph/0303394

Beers, T. C., Flynn, K., \& Gebhardt, K. 1990, AJ, 100, 32

Benitez, N. 2000, ApJ, 536, 571

Bertin, E., \& Arnouts, S. 1996, A\&AS, 117, 393

Bunker, A. J., Stern, D., Spinrad, H., Dey, A., Steidel, C. C. 1998, BAAS, 30, 927

Capak, P., et al. 2003, AJ, in press, astro-ph/0312635

Capak, P., et al. 2004, in preparation

Cohen, J.G., Cowie, L. L., Hogg, D. W., Songaila, A., Blandford, R., Hu, E. M., \& Shopbell, P. 1996, ApJ, 471, L5

Cohen, J.G., Hogg, D. W., Blandford, R., Cowie, L. L., Hu, E. M., Songaila, A., Shopbell, P. \& Richberg, K. 2000, ApJ, 538, 29

Cohen, J. G. 2001, AJ, 121, 2895

Cohen, J. G. 2002, ApJ, 567, 672

Cowie, L. L., Songaila, A., Hu, E. M. \& Cohen, J. G. 1996, AJ, 112,839

Cowie, L. L., Barger, A. J., Hu, E. M. \& Capak, P. 2004, AJ, submitted

Coleman, G. D., Wu, C.-C., Weedman, D. W. 1980, ApJS, 43, 393

Davis, M., et al. (The DEEP2 Team) 2003, SPIE, 4834, 161

Dawson, S., Stern, D., Bunker, A. J., Spinrad, H., \& Dey, A. 2001, AJ, 122,598

Dickinson, M. 1998 in The Hubble Deep Field, ed. M. Livio, S. M. Fall, \& P. Madau (New York: Cambridge Univ. Press), 219

Dickinson, M., et al. 2001, BAAS, 33, 820
Dickinson, M., et al. 2004, in preparation

Faber, S. M., et al. 2003, SPIE, 4841, 1657

Ferguson, H. C.. Dickinson, M., \& Williams, R. 2000, ARA\&A, 38, 667

Giavalisco, M., et al. 2004, in preparation

Glazebrook, K., Offer, A. R., Deeley, K. 1998, ApJ, 492, 98

Hogg, D. W., et al. (The SDSS collaboration) 2002, AJ, 124, 646

Im, M., Simard, L., Faber, S. M., Koo, D. C., Gebhardt, K., Willmer, C. N. A., Phillips, A. C., Illingworth, G. D., Vogt, N. P., \& Sarajedini, V. L. 2002, ApJ, 571, 136

Kinney, A. L., Calzetti, D., Bohlin, R. C., McQuade, K., StorchiBergmann, T., \& Schmitt, H. R. 1996, ApJ, 467, 38

le Fèvre, O., Crampton, D., Lilly, S. J., Hammer, F., \& Tresse, L. 1995, ApJ, 455, 60

Lowenthal, J. D., Koo, D. C., Guzmán, R., Gallego, J., Phillips, A. C., Vogt, N. P., Faber, S. M., Illingworth, G. D., \& Gronwall, C. 1997, ApJ, 481, 673

Marleau, F. R., \& Simard, L. 1998, ApJ, 507, 585

Monet, D. G., et al. 2003, AJ, 125, 984

Moustakas, L. A., Zepf, S. E., \& Davis, M. 1997, in The Hubble Space Telescope and the High Redshift Universe, ed. N. Tanvir, A. Aragon-Salamanca, \& J. Wall (Singapore: World Scientific), 273

Newman, J. A., et al. (The DEEP2 Team) 2004, in preparation

Oke, J. B. 1974, ApJS, 27, 21

Oke, J. B., Cohen, J. G., Carr, M., Cromer, J., Dingizian, A., Harris, F. H., Labrecque, S., Lucinio, R., Schaal, W., Epps, H., \& Miller, J. 1995, PASP, 107, 135 
Phillips, A. C., Guzmán, R., Koo, D. C., Lowenthal, J. D., Vogt, N. P., Faber, S. M., \& Illingworth, G. D. 1997, ApJ, 489, 543

Simard, L., et al. 2002, ApJS, 142, 1

Smith, J. A., et al. (The SDSS collaboration) 2002, AJ, 123, 2121

Steidel, C. C., Giavalisco, M., Dickinson, M., \& Adelberger, K. L. 1996, AJ, 112, 352

Steidel, C. C., Adelberger, K. L., Shapley, A. E., Pettini, M., Dickinson, M., \& Giavalisco, M. 2003, ApJ, 592, 728

Springel, V., \& White, S. D. M. 1988, MNRAS, 298, 143

Strateva, I., et al. (The SDSS Collaboration) 2001, AJ, 122, 186
Strauss, M. A., et al. (The SDSS Collaboration) 2002, AJ, 124, 1810

Treu, T. et al. 2004, in preparation

Weiner, B., et al. (The DEEP2 Team) 2004, in preparation

Willmer, C. N. A., et al. (The DEEP2 Team) 2004, in preparation

York, D. G., et al. (The SDSS Collaboration) 2000, AJ, 120, 1579

Zepf, S. E., Moustakas, L. A., \& Davis, M. 1997, ApJ, 474, L1 
TABLE 6. TKRS CATAlog

\begin{tabular}{|c|c|c|c|c|c|c|c|c|c|c|c|c|c|c|c|c|c|c|}
\hline No. $^{\mathrm{a}}$ & $\begin{array}{c}\alpha^{\mathrm{b}} \\
(\mathrm{J} 2000)\end{array}$ & $\begin{array}{c}\delta^{\mathrm{c}} \\
(\mathrm{J} 2000)\end{array}$ & $R_{A B}{ }^{\mathrm{d}}$ & Mask $^{\mathrm{e}}$ & Slit $^{f}$ & $z^{\mathrm{g}}$ & $Q^{\mathrm{h}}$ & $z_{o}^{\mathrm{i}}$ & Ref. $^{j}$ & $\begin{array}{l}X_{d}^{\mathrm{k}} \\
(\mathrm{px})\end{array}$ & $\begin{array}{l}Y_{d}^{1} \\
(\mathrm{px})\end{array}$ & $\mathrm{ACS}^{\mathrm{m}}$ & $\begin{array}{l}X_{a}{ }^{\mathrm{n}} \\
(\mathrm{px})\end{array}$ & $\begin{array}{l}Y_{a}{ }^{\mathrm{o}} \\
(\mathrm{px})\end{array}$ & $\begin{array}{l}a^{\mathrm{P}} \\
\left({ }^{\prime \prime}\right)\end{array}$ & $e 2^{\mathrm{q}}$ & $\begin{array}{l}\Theta^{\mathrm{r}} \\
\left({ }^{\circ}\right)\end{array}$ & ACS No \\
\hline 1468 & 123618.450 & 621601.53 & 23.18 & 01 & 046 & 0.79803 & 4 & 0.7970 & 20 & 3291.7 & 466.3 & 43 & 4574.6 & 7529.9 & 0.521 & 0.008 & 63.9 & J123618.46+62 1601.9 \\
\hline 1473 & 123601.805 & $62 \quad 1405.19$ & 24.20 & 01 & 025 & 0.43592 & 4 & $\ldots$ & $\cdots$ & 1897.3 & 462.3 & 53 & 272.6 & 3663.6 & 0.674 & 0.225 & 133.4 & $\mathrm{~J} 123601.80+621405.6$ \\
\hline 1475 & 123605.932 & 621435.81 & 22.16 & 01 & 029 & 0.40836 & 4 & $\ldots$ & $\cdots$ & 2253.8 & 452.9 & 43 & 7490.8 & 4678.5 & 0.636 & 0.028 & 19.2 & $\mathrm{~J} 123605.97+621436.1$ \\
\hline 1479 & 123625.632 & $62 \quad 1647.94$ & 24.61 & $\cdots$ & $\ldots$ & $\ldots$ & $\cdots$ & $\cdots$ & $\cdots$ & 3870.3 & 489.7 & 44 & 2897.1 & 886.6 & 0.372 & 0.003 & -34.8 & J123625.65+6 1648.3 \\
\hline 1482 & 123700.626 & $62 \quad 20 \quad 54.44$ & 23.79 & 02 & 096 & 0.79390 & 4 & $\cdots$ & $\cdots$ & 6809.1 & 473.9 & 35 & 2969.6 & 904.3 & 0.572 & 0.009 & 117.4 & $\mathrm{~J} 123700.59+\underset{2}{2} 054.9$ \\
\hline
\end{tabular}

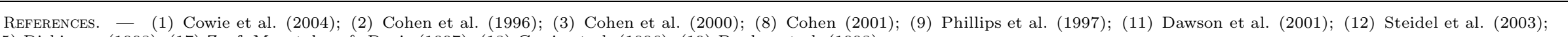
(15) Dickinson (1998); (17) Zepf, Moustakas, \& Davis (1997); (18) Cowie et al. (1996); (19) Bunker et al. (1998).

NoтE. - Table 6 is published in its entirety in the electronic edition of The Astronomical Journal. A portion is shown here for guidance regarding its form and content.

aSerial number in TKRS catalog.

Right Ascension, derived from DEIMOS mosaic image.

${ }^{c}$ Declination, derived from DEIMOS mosaic image.

$R$ magnitude in $\mathrm{AB}$ system, derived from DEIMOS mosaic image.

TKRS mask number with which object was observed.

${ }^{\text {f}}$ Slit number for object on TKRS mask.

Topocentric redshift measured by TKRS.

i Redshift code assigned by TKRS

jSource of alternate redshift.

${ }^{\mathrm{k}} \mathrm{X}$ position on the DEIMOS mosaic image.

Y position on the DEIMOS mosaic image.

${ }^{\mathrm{m}}$ Sector number in which the object lies on the HST/ACS mosaic image.

${ }^{\mathrm{n} X}$ position on the HST/ACS mosaic image.

oY position on the HST/ACS mosaic image.

PSemi-major axis length measured by SExtractor on DEIMOS mosaic image.

"Ellipticity measured by SExtractor on DEIMOS mosaic image.

${ }^{\mathrm{r}}$ Major axis position angle measured by SExtractor on DEIMOS mosaic image $($ North $=0$, East $=+90)$.

${ }^{s}$ GOODS-N v1.0 catalog IAU identification of objects matching the DEIMOS catalogue from Giavalisco et al. (2004). 